\title{
Development of Conductive Polymer Analysis for the Rapid Detection and Identification of Phytopathogenic Microbes
}

\author{
A. D. Wilson, D. G. Lester, and C. S. Oberle
}

U.S. Department of Agriculture, Forest Service, Forest Insect and Disease Research, Southern Research Station, Center for Bottomland Hardwoods Research, Southern Hardwoods Laboratory, 432 Stoneville Road, Stoneville, MS 38776. Accepted for publication 12 January 2004.

\section{ABSTRACT}

Wilson, A. D., Lester, D. G., and Oberle, C. S. 2004. Development of conductive polymer analysis for the rapid detection and identification of phytopathogenic microbes. Phytopathology 94:419-431.

Conductive polymer analysis, a type of electronic aroma detection technology, was evaluated for its efficacy in the detection, identification, and discrimination of plant-pathogenic microorganisms on standardized media and in diseased plant tissues. The method is based on the acquisition of a diagnostic electronic fingerprint derived from multisensor responses to distinct mixtures of volatile metabolites released into sampled headspace. Protocols were established to apply this technology specifically to plant disease diagnosis. This involved development of standardized cultural methods, new instrument architecture for sampling, sample preparation, prerun procedures, run parameters and schedules, recognition files and libraries, data manipulations, and validation protocols for interpretations of results. The collective output from a 32-sensor array pro- duced unique electronic aroma signature patterns diagnostic of individual microbial species in culture and specific pathogen-host combinations associated with diseased plants. The level of discrimination applied in identifications of unknowns was regulated by confidence level and sensitivity settings during construction of application-specific reference libraries for each category of microbe or microbe-host combination identified. Applications of this technology were demonstrated for the diagnosis of specific disease systems, including bacterial and fungal diseases and decays of trees; for host identifications; and for determinations of levels of infection and relatedness between microbial species. Other potential applications to plant pathology are discussed with some advantages and limitations for each type of diagnostic application.

Additional keywords: artificial neural network, artificial olfaction, bacterial wetwood, early detection, electronic nose, homeland security, oak wilt.
Recent advances in biochemistry, sensor technology, electronics, and artificial intelligence have made it possible to develop devices capable of measuring and characterizing volatile aromas or odors, produced by a multitude of sources, for numerous applications. These devices, known as electronic noses, were developed in an attempt to mimic a mammalian olfactory system within an instrument that obtains repeatable digital measurements, allowing determinations of aroma identifications or classifications while eliminating operator fatigue $(6,9,29-31,38,39)$. The technology of artificial olfaction had its beginning with the invention of the first gas multisensor array in 1982 (9), but use of the term "electronic nose" for instruments made with this technology was not coined until 1988. Since then, more than a hundred different prototypes of artificial nose devices have been developed to discriminate aromatic mixtures containing many different types of volatile organic compounds $(27,50)$. The prototypes represent several types of electronic aroma detection (EAD) technologies that utilize different sensor types, including metal-oxides $(11,26,40)$, semiconductive polymers $(22,34,51)$, and conductive electroactive polymers $(12,17,21,33)$, that are capable of direct electronic detection and identification of complex mixtures in sampled air. Electronic noses originally were not designed for the purpose of identifying individual chemical species within the sample mixture, but were engineered to recognize the sample as a whole, or as a collective simple or complex air mixture released by any source that is identifiable by its unique electronic signature.

Corresponding author: A. D. Wilson; E-mail address: dwilson02@fs.fed.us

Publication no. P-2004-0311-01R

This article is in the public domain and not copyrightable. It may be freely reprinted with customary crediting of the source. The American Phytopathological Society, 2004
The typical electronic nose instrument consists of a multisensor array, an information-processing system such as an artificial neural network (ANN), software with digital pattern-recognition algorithms, and reference libraries to discriminate samples by their unique aroma signatures $(1,13,15,20,27)$. The sensor array is composed of incrementally different sensors chosen to respond to a wide range of different chemicals or chemical classes. This sensor architecture is capable of discriminating diverse mixtures of possible analytes. Each sensor in the array produces an output that, when assembled collectively, forms a distinct pattern of responses (digital fingerprint) called an electronic aroma signature pattern (EASP), which allows classification and identification of the analyte. In this way, the array of sensors is integrated to yield a unique signal for a complex of distinctive aromas without requiring the mixture to be separated into its individual components prior to or during analysis. To use the instrument, a reference library of known EASPs must first be constructed by assigning descriptor names (identifiers) to patterns of known origin. The ANN is configured through a learning process (neural-net training) using pattern recognition algorithms that look for differences between the patterns of all the descriptor types to be included in the reference library. This process continues until a previously specified level of discrimination (determined by preset parameters) is met. The results from these comparisons are validated and then collectively assembled into the reference library to which unknown samples can be compared for classification and identification based on the distribution of aroma attributes or elements that the analyte pattern has in common with patterns present in the databases of the reference library.

Most applications of EAD technology hitherto have been in industrial food production, processing, and manufacturing $(2,3$, $10,16,27,33,34)$. Some of the more common applications have 
been in the areas of quality control and grading, detection of microbial contamination in food products, food freshness and storage, gas leak detection, processing controls, and environmental monitoring $(7,8,27,32,41,43,47,50)$. One notable example is the routine use of EAD technology by the U.S. Department of Agriculture Food Safety and Inspection Service for quality control and detection of contaminated meat. More recently, EAD has been used in the biomedical field for applications in medical diagnostics such as detecting human pathogens in diseased tissues $(19,28,44)$, microbial mensuration (14), and waste management $(18,32)$. Using EAD technology as a means for identifying microbial species that cause diseases of plants is quite different from other approaches that accomplish this by identifying diagnostic structural compounds found in the cell walls or by sequencing genomic or extragenomic DNA or RNA of the microbes themselves $(4,23-25,35,36)$.

This article reports on the development of conductive polymer analysis (CPA) for the rapid identification and discrimination of phytopathogenic microbes based on their production of unique mixtures of volatile metabolites recorded as diagnostic EASPs. The composition of primary and secondary metabolites released by individual microbes is controlled largely by the types and combinations of metabolic pathways specific to microbial species. These metabolic pathways are regulated by genetic, substrate, and environments factors. Differences in types and proportions of metabolic products released by different strains within a species are determined primarily by genetic differences when strains are grown under identical conditions on a standardized culture medium. The objectives of this study were to (i) evaluate the feasibility of using CPA as a means of detecting, identifying, and discriminating the presence of plant-pathogenic microbes in pure culture and in host tissues; (ii) develop and test means and protocols for applying CPA technology to plant disease diagnosis; and (iii) determine some of the potential applications of this technology to plant pathology. Preliminary results of this work were reported previously $(48,49)$.

\section{MATERIALS AND METHODS}

Collection and culture of microbes. A number of microbial strains, used here to construct reference libraries of EASP databases, were isolated from a variety of plant hosts (Table 1). Most strains of phytopathogenic bacteria and fungi were obtained from the American Type Culture Collection (Manassas, VA). Some bacterial strains were acquired as a gift from D. Gross (Texas A\&M University, College Station). A few fungal strains were obtained from D. Ingram (Central Mississippi Research and Extension Center, Raymond, MS), J. Bruhn (University of Missouri, Columbia) from isolations in Ozark Mountain oak forests (5), F. Tainter (Clemson University, Clemson, SC), and D. Appel (Texas A\&M University). Strains of wood-decay fungi were isolated from contextual tissues of freshly collected basidiocarps on $4.5 \%$ malt agar (MA) with $0.1 \%$ streptomycin sulfate.

All microbes within each category (bacteria or fungi) were grown under identical cultural conditions on a universally favorable standardized culture medium. Strains of plant-pathogenic and wood-decay fungi were cultured on $4.5 \%$ MA. Phytopathogenic bacteria were cultured on nutrient-broth yeast extract agar (NBY), prepared according to Vidaver (45). Slant cultures were prepared in screw-capped glass tubes $(20$ by $70 \mathrm{~mm}$ ) with approximately $7 \mathrm{ml}$ of growth medium and incubated at $25^{\circ} \mathrm{C}$ (fungi) or $30^{\circ} \mathrm{C}$ (bacteria) until growth had covered the entire agar surface, approximately $665 \mathrm{~mm}^{2}$.

Collection and storage of diseased tissue samples. All of the diseased plant samples collected in this study were taken from woody plants using a Haglöf tree increment borer (Forest Suppliers, Inc., Jackson, MS). Tree cores of standard dimensions ( $5 \mathrm{~mm}$ in diameter by $5 \mathrm{~cm}$ in length) were collected from either heartwood, sapwood, or both of individual trees, depending on where the diseased or colonized tissues were located. Extracted cores were placed either in plastic straws with bent and taped ends or in 14.8-mm glass vials used to place samples into the sampling bottle. The cores were frozen at $-20^{\circ} \mathrm{C}$ in long-term storage and thawed immediately prior to sample analysis. Cores that became desiccated due to sublimation during storage were rehydrated by soaking in sterile distilled water for $15 \mathrm{~min}$ followed by blotting on Chemwipe tissue paper to remove excess moisture immediately prior to analysis.

Sample preparation and prerun procedures. Sample cultures in 14.8-ml glass vials were uncapped and placed into a 500-ml glass sampling bottle fitted with reference air, sampling, and exhaust ports on a polypropylene bottle cap. Reference air entered the sampling bottle through a 3-mm polypropylene tube extending to just above the bottom of the sampling bottle. The sampling bottle was held in the sampling chamber within the instrument at a constant air temperature of $25^{\circ} \mathrm{C}$. The sampling bottle was purged with filtered, moisture-conditioned reference air for 2 min prior to building headspace. The sampling bottle was sealed and volatiles from the sample were allowed to build headspace and equilibrate for $30 \mathrm{~min}$ prior to each run. Prerun tests were performed as needed to determine sample air relative humidity $(\mathrm{RH})$ compared with that of reference air. Reference air was set at $4 \%$ $\mathrm{RH}$ for most runs and adjusted to within $2 \%$ below sample air at $25^{\circ} \mathrm{C}$. The sampling bottle cap and exhaust port were opened between runs to purge the previous sample with conditioned reference air.

Instrument configuration and run parameters. All analyses were conducted with an Aromascan A32S (Osmetech, Inc., Wobum, MA) instrument fitted with a conventional 32-sensor array designed for general-use applications with $15 \mathrm{~V}$ across sensor paths. Prior to analysis of known and unknown analytes in this study, individual sensors in the sensor array were characterized by testing sensitivity responses to representative compounds from nine classes of organic compounds potentially relevant to microbial identifications. The response sensitivities of individual sensors, measured in percent as relative differential electricalresistance changes $\left(\% \Delta \mathrm{R} / \mathrm{R}_{\text {base }}\right)$, varied with the type of plastic polymer used in the sensor matrix coating, the type of ring-substitution used to modify its conductive properties, and the type of metal ions used to dope the matrix to improve and modulate sensor response (Table 2). The detection limits of the instrument were dependent on the classes of organic components present in the sample and the combined sensitivities of the sensor array. The sensors generally were most sensitive to amines and sulfur-containing compounds. However, highly polar compounds like carboxylic acids tended to bind and accumulate on some sensors, causing negative responses in some cases at higher concentrations.

The block temperature of the sensor array was maintained at a constant $30^{\circ} \mathrm{C}$. Reference air was preconditioned by passing room air sequentially through a carbon filter, silica gel beads, inline filter, and Hepa filter to remove organic compounds, moisture, particulates, and microbes, respectively, prior to humidity control and introduction into the sampling bottle. The flow rate (suction) of sample air at the sampling port was maintained at $-702 \mathrm{ml} / \mathrm{min}$ using a calibrated ADM 3000 flow meter (Agilent Technologies, Wilmington, DE). Sensors were purged between runs using a $2 \%$ isopropanol wash solution. The instrument was interfaced with a personal computer via a RS232 cable and controlled with Aromascan (version 3.51) software. The instrument plumbing was altered from conventional architecture and specifically configured for static sampling of the headspace by allowing air flow, maintained at $605 \mathrm{ml} / \mathrm{min}$ flow rate, coming out of the external vent (bag-fill) port of the instrument during analytical runs, and closing the exhaust port on the sampling bottle so that headspace volatiles were removed from a homogeneous static air mass within the sampling bottle. 
Data acquisition parameters and run schedules. Data from the sensor array were collected at 1-s intervals using a 0.2 detection threshold ( $y$ units), a 15- to 20-y-max graph scale, and a pattern average of five data samples taken per run during data acquisition. A uniform run schedule (one valve sequence) was used and consisted of reference air, $20 \mathrm{~s}$; sampling time, $90 \mathrm{~s}$; and wash, $20 \mathrm{~s}$; followed by $90 \mathrm{~s}$ of reference air; for a total run time of $220 \mathrm{~s}$. A 2-min reference air purge followed by a 30-min equilibration period was allowed between runs.

Construction of reference libraries and validation. Separate reference libraries were constructed for each specific category of microbes (bacteria and fungi) from known reference strains of each species included in each library. All database files of reference strains were linked to specific (designated) aroma classes for each sample type. A separate neural-net training session was conducted to create a unique reference library applicable for each microbe category analyzed. A minimum of 10 replicate runs per strain and multiple strains ( 2 to 10 per species) were used to construct each reference library. The following recognition network options (neural-net training parameters) generally were used for each training session: training threshold $=0.60$, recognition threshold $=0.60$, number of elements allowed in error $=5$, learning rate $=0.10$, momentum $=0.60$, error goal $=0.010(P \leq 0.01)$, hidden nodes $=5$, and maximum iterations (epochs) $=10,000$, using normalized input data, not actual intensity data. Some of these parameters were modified as described in the results for specific applications or for improvement of recognition accuracy. A typical neural-net training required 2 to $35 \mathrm{~min}$, depending on the size of the database applied, using an IBM-compatible personal computer with a minimum of $64 \mathrm{MB}$ of RAM and $350 \mathrm{MHz}$ run speed. Neural net trainings were validated by examining training results that compared individual database files for compatibility with its defined aroma class, and displayed similarity matches using aroma class distributions (percentages) among all aroma classes included in the recognition file, created by the training.

Identification of unknowns using recognition files. Reference libraries of signature patterns of headspace volatiles from known microbes were used for identification of unknown samples. This was accomplished using a recognition file (that defines each reference library) either in real time or in offline analysis. In either case, the neural net compared the response pattern of the unknown sample with databases found in the reference library chosen for comparison. The recognition algorithms quickly determined the best match that most closely fit the aroma elements found in the unknown sample. The closeness of the match was expressed as a percentage value assigned to different global

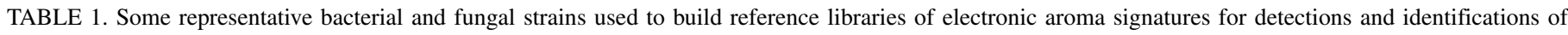
microbes isolated on standardized culture media

\begin{tabular}{|c|c|c|c|c|}
\hline Microbe & Strain $^{\mathrm{a}}$ & Host origin & Disease & Location \\
\hline \multicolumn{5}{|l|}{ Bacteria } \\
\hline Brenneria quercina & ATCC 29281 & Quercus agrifolia & Drippy nut & California \\
\hline Clavibacter michiganensis subsp. michiganensis & $15-2$ & Lycopersicon esculentum & Bacterial canker & North Carolina \\
\hline C. michiganensis subsp. nebraskensis & ATCC 27794 & Zea mays & Corn stunting & Nebraska \\
\hline Curtobacterium flaccumfaciens var. violaceum & CV6 & Phaseolus vulgaris & Bacterial wilt & Nebraska \\
\hline \multirow[t]{2}{*}{ Erwinia amylovora } & E9 & Pyrus communis & Fire blight & Idaho \\
\hline & ATCC 15580 & P. communis & Fire blight & United Kingdom \\
\hline E. chrysanthemi & A310 & Saintpaulia ionantha & Bacterial blight & Idaho \\
\hline Pantoea ananatis & Eh112Y & Malus domestica & Root galls & United Kingdom \\
\hline $\begin{array}{l}\text { Pectobacterium carotovora subsp. atroseptica } \\
(=\text { Erwinia carotovora subsp. atroseptica) }\end{array}$ & W3C431 & Solanum tuberosum & Black leg & Washington \\
\hline \multirow{9}{*}{$\begin{array}{l}\text { P. carotovora subsp. carotovora } \\
\quad(=\text { Erwinia carotovora subsp. carotovora) } \\
\text { Pseudomonas putida } \\
\text { P. syringae pv. morsprunorum } \\
\text { P. syringae pv. syringae } \\
\text { Ralstonia solanacearum } \\
\text { Rhizobium rhizogenes (=Agrobacterium rhizogenes) } \\
R \text {. radiobacter (=Agrobacterium tumefaciens) }\end{array}$} & W3C827 & S. tuberosum & Soft rot & Washington \\
\hline & ATCC 15713 & S. tuberosum & Soft rot & Denmark \\
\hline & W4P5 & S. tuberosum & Root saprophyte & Washington \\
\hline & ATCC 13395 & Prunus avium & Leaf spot & New Zealand \\
\hline & B-3A & P. persica & Bacterial canker & California \\
\hline & ATCC 11696 & L. esculentum & Southern wilt & North Carolina \\
\hline & ATCC 11325 & M. domestica & Hairy root & Wisconsin \\
\hline & NCPPB 2437 & L. esculentum & Crown gall & United States \\
\hline & ATCC 23308 & P. persica & Crown gall & United States \\
\hline Rhodococcus fascians & ICPB CF107 & $\ldots$ & Fasciations & United States \\
\hline Streptomyces scabies & ATCC 49173 & S. tuberosum & Common scab & New York \\
\hline \multirow{2}{*}{$\begin{array}{l}\text { Xanthomonas arboricola } \mathrm{pv} . \text { juglandis } \\
\quad(=X . \text { juglandis } \mathrm{pv} . \text { juglandis })\end{array}$} & ATCC 11329 & Juglans regia & Bacterial blight & United States \\
\hline & $\mathrm{W} 5 \mathrm{C} 1$ & J. regia & Bacterial blight & Washington \\
\hline$X$. fragariae & ATCC 33239 & Fragaria chiloensis & Angular leaf spot & United States \\
\hline X. oryzae pv. oryzae & ATCC 35933 & Oryza sativa & Leaf blight & India \\
\hline \multicolumn{5}{|l|}{ Fungi } \\
\hline Armillaria mellea & JNB-OZ298 & Quercus velutina & Root rot & Missouri \\
\hline A. tabescens & JNB-MOAB1 & Q. rubra & Root rot & Missouri \\
\hline Ceratocystis fagacearum & ADW-TX15 & Q. fusiformis & Oak wilt & Texas \\
\hline Cryphonectria parasitica & FHT-SC1 & Castanea dentata & Chestnut blight & South Carolina \\
\hline Daedalea quercina & ADW-930103 & Q. nuttallii & Bole rot & Mississippi \\
\hline Ganoderma lucidum & ADW-962501 & Carya illinoensis & Root and butt rot & Mississippi \\
\hline Hericium erinaceus & ADW-922204 & Q. nigra & Bole rot & Mississippi \\
\hline Inonotus dryadeus & ADW-993001 & Q. phellos & Bole rot & Mississippi \\
\hline Laetiporus sulphureus & ADW-971801 & Q. nuttallii & Butt rot & Mississippi \\
\hline Ophiostoma piceae & DNA-TX1 & Pinus sp. & Endophyte & Minnesota \\
\hline O. pluriannulatum & DNA-TX2 & Quercus sp. & Endophyte & Minnesota \\
\hline Pleurotus ostreatus & ADW-970308 & Salix nigra & Butt rot & Mississippi \\
\hline Pythium graminicola & DMI-WA1 & T. aestivum & Root rot & Washington \\
\hline P. oligandrum & DMI-WA2 & T. aestivum & Root rot & Washington \\
\hline P. ultimum sporangiferum & DMI-WA3 & T. aestivum & Root rot & Washington \\
\hline Schizophyllum commune & ADW-970305 & Q. falcata & Bole rot & Mississippi \\
\hline Trametes versicolor & ADW-970301 & Q. falcata & Bole rot & Mississippi \\
\hline
\end{tabular}

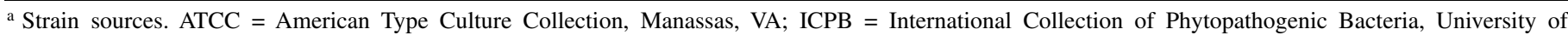
California, Davis; NCPPB = National Collection of Plant-Pathogenic Bacteria, Central Science Laboratory, Sand Hutton, York, UK. 
classes represented in the sample. A value greater than $95 \%$ was considered to be a good match. Once separate reference databases were developed for bacteria and fungi growing on their respective growth medium, unknowns were identified using separate recognition files linked to a specific reference library. This was accomplished by specifying a recognition file, linked to the specific reference library selected for comparison, in the data acquisition window. This additional specification was added and saved as a separate run method from that used for scans of known samples used in building reference libraries for specific types of microbes. The neural net software had settings that allowed for training to any level of significance that was desired in discriminating samples. A significance level of 0.05 or lower was used in these applications depending on the level of specificity desired in the discrimination.

Detection of infection status in living tissues. Headspace volatiles from living sapwood cores from the outer sapwood of live oaks were used to test the ability of CPA to diagnose oak wilt in living trees at various stages of infection. Mean global class distributions provided indications of the percentages of aroma elements in the unknown sample that matched those in the reference library. Three aroma global classes (healthy, infected, and unknown) were defined in the reference library to represent the infection status categories for classifying these unknown samples.

Instrument reliability in identifications. The reliability of the instrument and methods to accurately identify sample unknowns was tested with double-blind tests. Analyzed samples were determined to be either correctly identified, not identified (indetermi- nate), incorrectly identified, or ambiguously identified. Determinations falling outside of the domain of defined global classes were recorded as unknown. Ambiguous determinations resulted when different strains of a given microbial species were identified in different global classes from separate runs.

Data processing, manipulations, and statistical evaluations. Data slices for processing and analysis were taken from a conventional 20-s sampling interval ( 85 to $105 \mathrm{~s}$ ) near the end of the sampling segment of each run, before the sampling-valve closed. The data slice from the raw data file was used to create a representative descriptor database file. A minimum of 10 descriptor database files were created from separate cultures of each microbial strain or sample type. Aroma signature patterns of individual aroma classes (descriptors) were reported from calculated means \pm standard errors of the mean of raw relative resistance sensor values from 10 replicate runs of 2 to 10 different samples for each aroma class. Real-time determinations of unknowns utilized recognition files with normalized sensor intensity responses and pattern recognition algorithms and matrices.

Detailed comparisons of relatedness of aroma classes were determined using principal component analysis (PCA) algorithms provided in the Aromascan (version 3.51) software. Three-dimensional PCA was used to distinguish between headspace volatiles released from related species of Ceratocystis and Ophiostoma fungi on MA culture medium, and between headspace volatiles released from live oak sapwood tree cores at various levels of infection by Ceratocystis fagacearum, the oak wilt fungus, for disease diagnosis. The mapping parameters for three-dimensional PCA were as follows: iterations $=30$, units in Eigen values $(\%)$,

TABLE 2. Relative sensitivity responses of individual sensors in the Aromascan A32S sensor array to representative members of nine classes of organic compounds $^{\mathrm{a}}$

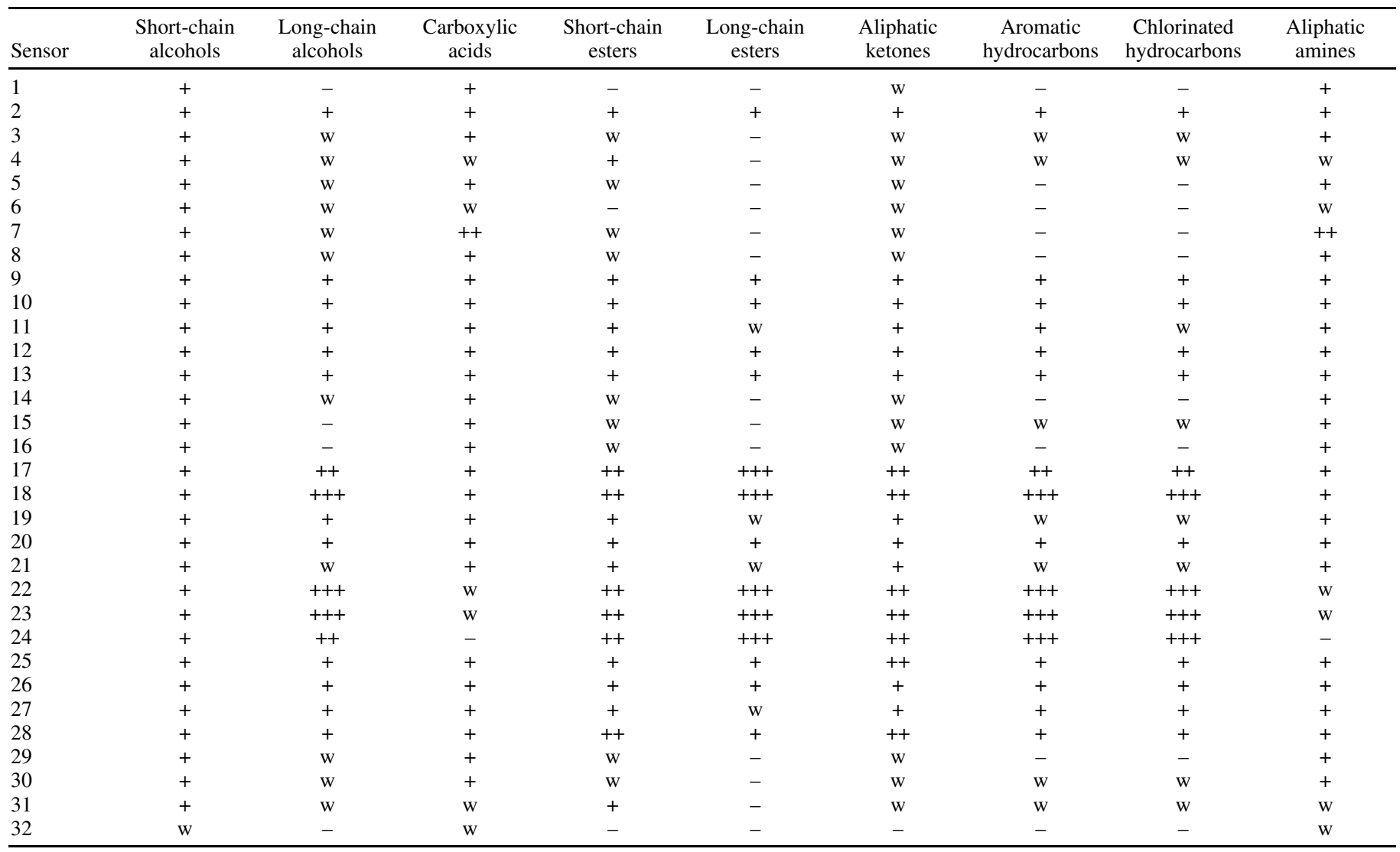

${ }^{a}$ Sensor sensitivities defined by electrical resistance response, percentage deviations ( $\left.\% \Delta \mathrm{R} / \mathrm{R}_{\text {base }}\right)$ across sensor paths relative to base resistances, produced by adsorption and interaction of analytes (odorants) from each specified chemical class, to the surface of the sensors, according to the following scale: $+++=$ very strong $(R>9 \%) ;++=$ strong $(5 \%<R<9 \%) ;+=$ moderate $(2 \%<R<5 \%)$; w $=$ trace to weak $(1 \%<R<2 \%)$; and $-=$ negligible $(R<1 \%)$ response. Sensors in the array are coated with conductive polymers produced by electropolymerization of either polypyrrole, polyanaline, or polythiophene derivatives which have been modified with ring-substitutions of different functional groups that impart unique conductive properties (electrical resistance responses to different classes of compounds). These polymers are doped with specific metal ions to improve and modulate polymer conductivity and sensor sensitivity. 
and with normalized input data. Two-dimensional Sammon mapping analysis (SMA) of headspace volatiles released from cultures of five bacterial species, formerly placed in the genus $\mathrm{Er}$ winia and grown on standardized NBY growth medium, was used to construct aroma maps for comparisons of relatedness of aroma classes and indications of taxonomic relatedness. The mapping parameters utilized for two-dimensional Sammon mapping were as follows: maximum iterations $=100$, minimum error $=0.001$, scaling factor $=2.0$, using normalized input data.

\section{RESULTS}

Run characteristics and pattern analysis of microbial species. Unique EASPs were produced by CPA of headspace volatiles from cultures of phytopathogenic bacteria and fungi. Noticeable differences were observed between the A32S multisensor outputs and run characteristics for these two types of microbes during data acquisition. Headspace volatiles from plantpathogenic fungi produced relatively flat, low-resistance response curves $(<5 \%$ above baseline electrical resistance of each sensor) with tightly clustered outputs of individual sensors (Fig. 1A). Runs of volatiles from bacteria produced multisensor outputs that were much more diffuse, and with higher intensity responses (up to $15 \%$ or more) above baseline resistances (Fig. 1B). Comparisons of normalized sensor outputs as histograms indicated low variability and lower sensor responses to volatiles from fungi compared with the generally stronger intensity and more variable sensor responses to volatiles of bacteria (Fig. 1C and D). The greater variability and stronger sensor-response intensities to bacteria relative to fungi were more evident when comparing EASPs of pathogenic bacteria and fungi from many host sources and locations (Table 3). Statistical analysis showed high precision and low variability of individual sensor responses between analytical runs for individual strains and between sympatric strains within microbial species. However, the variability of aroma profiles among strains within species was greater with allopatric strains from widely separated localities. Headspace volatiles of exceptional bacteria, such as Pantoea ananatis, Pseudomonas putida, Rhizobium rhizogenes, and Streptomyces scabies that produced sensor intensity responses of $>10 \%$ for multiple sensors within

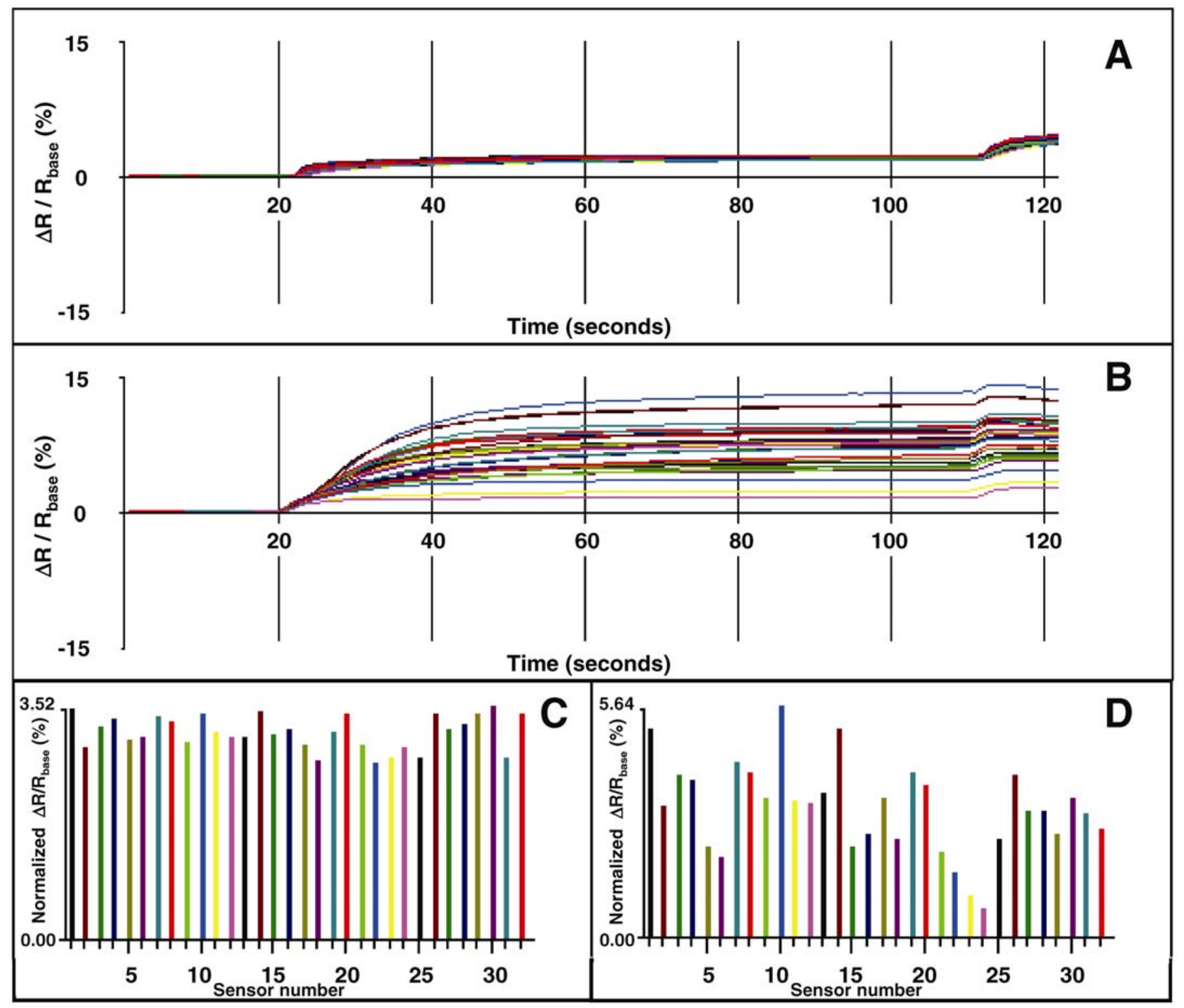

Fig. 1. Analytical outputs of the Aromascan A32S sensor array produced from conductive polymer analyses of headspace volatile metabolites from representative species of fungal and bacterial microbes on standardized growth media. A, Clustered multisensor output from a typical fungal pathogen (Ceratocystis fagacearum shown), the oak wilt fungus. B, Diffuse multisensor output from a typical bacterial pathogen (Rhizobium rhizogenes shown). Normalized intensity responses of individual sensors $\left(\% \Delta \mathrm{R} / \mathrm{R}_{\text {base }}\right)$ to headspace volatiles produced by $\mathbf{C}, C$. fagacearum and $\mathbf{D}, R$. rhizogenes. 


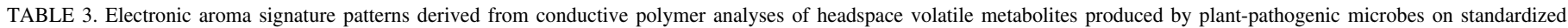
culture media

\begin{tabular}{|c|c|c|c|c|c|c|c|c|c|c|c|c|c|c|c|}
\hline \multirow[b]{2}{*}{ Microbe $^{\mathrm{a}}$} & \multicolumn{15}{|c|}{ Sensor number $^{\mathrm{b}}$} \\
\hline & 1 & 2 & 3 & 4 & 5 & 6 & 7 & 8 & 9 & 10 & 11 & 12 & 13 & 14 & 15 \\
\hline \multicolumn{16}{|l|}{ Bacteria } \\
\hline B. quercina & $3 \pm 0.1$ & $8 \pm 0.1$ & $3 \pm 0.1$ & $2 \pm 0.2$ & $3 \pm 0.1$ & $1 \pm 0.1$ & $4 \pm 0.1$ & $3.2 \pm 0.1$ & $2.9 \pm 0.1$ & $4.0 \pm 0.1$ & $2.9 \pm 0.1$ & $2.7 \pm 0.1$ & $2.9 \pm 0.1$ & $3.7 \pm 0.1$ & $1.9 \pm 0.1$ \\
\hline C. michiganensis & $3.7 \pm 0.6$ & $.7 \pm 0.4$ & $2 \pm 0.5$ & $3.2 \pm 0.4$ & $2 \pm 0.2$ & $0 \pm 0.2$ & $4 \pm 0.5$ & $3.2 \pm 0.5$ & $2.8 \pm 0.4$ & $3.9 \pm 0.7$ & $2.8 \pm 0.4$ & $2.6 \pm 0.4$ & $2.8 \pm 0.4$ & $3.6 \pm 0.6$ & $1.9 \pm 0.2$ \\
\hline C. flaccumfaciens & $9.3 \pm 0.2$ & $5.7 \pm 0.2$ & $7.1 \pm 0.3$ & $6.9 \pm 0.2$ & $0 \pm 0.1$ & $.5 \pm 0.2$ & $7.8 \pm 0.4$ & $7.3 \pm 0.4$ & $6.1 \pm 0.3$ & $10.5 \pm 0.3$ & $6.0 \pm 0.2$ & $5.8 \pm 0.2$ & $6.2 \pm 0.2$ & $9.3 \pm 0.3$ & $3.6 \pm 0.1$ \\
\hline E. amylovora & $2.6 \pm 0.1$ & $2.1 \pm 0.1$ & $2.3 \pm 0.1$ & $2.3 \pm 0.1$ & $9 \pm 0.1$ & $.8 \pm 0.1$ & $2.4 \pm 0.1$ & $2.3 \pm 0.1$ & $2.1 \pm 0.1$ & $2.5 \pm 0.2$ & $2.1 \pm 0.1$ & $1.9 \pm 0.1$ & $2.1 \pm 0.1$ & $2.3 \pm 0.1$ & $1.5 \pm 0.1$ \\
\hline E. chrysanthemi & $1.7 \pm 0.1$ & $1.3 \pm 0.1$ & $1.6 \pm 0.1$ & $1.6 \pm 0.1$ & $.1 \pm 0.0$ & $.0 \pm 0.1$ & $1.7 \pm 0.1$ & $1.6 \pm 0.1$ & $1.4 \pm 0.0$ & $1.9 \pm 0.1$ & $1.3 \pm 0.1$ & $1.2 \pm 0.1$ & $1.2 \pm 0.1$ & $1.5 \pm 0.1$ & $0.8 \pm 0.1$ \\
\hline P. ananatis & $12.1 \pm 0.2$ & $7.5 \pm 0.2$ & $9.3 \pm 0.2$ & $9.0 \pm 0.3$ & $.2 \pm 0.2$ & $6 \pm 0.1$ & $10.1 \pm 0.2$ & $9.5 \pm 0.2$ & $7.9 \pm 0.2$ & $13.3 \pm 0.3$ & $7.8 \pm 0.2$ & $7.8 \pm 0.3$ & $8.1 \pm 0.1$ & $11.8 \pm 0.2$ & $5.1 \pm 0.2$ \\
\hline P. carotovora & $4.0 \pm 0.5$ & $2.9 \pm 0.3$ & $3.4 \pm 0.4$ & $3.4 \pm 0.3$ & $.5 \pm 0.2$ & $.3 \pm 0.1$ & $3.7 \pm 0.4$ & $3.5 \pm 0.4$ & $3.0 \pm 0.3$ & $4.1 \pm 0.5$ & $3.0 \pm 0.3$ & $2.8 \pm 0.3$ & $3.1 \pm 0.3$ & $3.8 \pm 0.5$ & $2.1 \pm 0.2$ \\
\hline P. putida & $11.4 \pm 0.4$ & $7.0 \pm 0.3$ & $8.8 \pm 0.4$ & $8.4 \pm 0.4$ & $4.5 \pm 0.3$ & $4.0 \pm 0.1$ & $9.4 \pm 0.4$ & $8.9 \pm 0.3$ & $7.4 \pm 0.2$ & $12.8 \pm 0.4$ & $7.2 \pm 0.3$ & $7.2 \pm 0.2$ & $7.7 \pm 0.2$ & $11.4 \pm 0.4$ & $4.6 \pm 0.2$ \\
\hline P. syringae & $1.8 \pm 0.1$ & $1.7 \pm 0.1$ & $1.8 \pm 0.1$ & $1.9 \pm 0.1$ & $0 \pm 0.1$ & $.0 \pm 0.1$ & $1.9 \pm 0.1$ & $1.9 \pm 0.1$ & $1.7 \pm 0.1$ & $1.6 \pm 0.1$ & $1.8 \pm 0.1$ & $1.7 \pm 0.1$ & $1.7 \pm 0.1$ & $1.6 \pm 0.1$ & $1.8 \pm 0.1$ \\
\hline R. solanacearum & $7.4 \pm 0.3$ & $4.8 \pm 0.1$ & $5.9 \pm 0.2$ & $5.7 \pm 0.1$ & $.5 \pm 0.2$ & $3.2 \pm 0.1$ & $6.4 \pm 0.1$ & $6.0 \pm 0.1$ & $5.1 \pm 0.0$ & $8.4 \pm 0.3$ & $5.0 \pm 0.1$ & $4.8 \pm 0.1$ & $5.2 \pm 0.2$ & $7.5 \pm 0.1$ & $3.0 \pm 0.2$ \\
\hline$R$. rhizogenes & $11.6 \pm 0.3$ & $7.3 \pm 0.2$ & $9.1 \pm 0.3$ & $8.8 \pm 0.2$ & $0 \pm 0.1$ & $.5 \pm 0.1$ & $9.8 \pm 0.2$ & $9.3 \pm 0.2$ & $7.8 \pm 0.3$ & $13.0 \pm 0.2$ & $7.6 \pm 0.3$ & $7.5 \pm 0.1$ & $8.0 \pm 0.0$ & $11.7 \pm 0.2$ & $5.0 \pm 0.1$ \\
\hline R. radiobacter & $2.8 \pm 0.4$ & $2.3 \pm 0.2$ & $2.6 \pm 0.3$ & $2.6 \pm 0.3$ & $2.2 \pm 0.1$ & $2.1 \pm 0.1$ & $2.7 \pm 0.3$ & $2.6 \pm 0.3$ & $2.4 \pm 0.2$ & $2.7 \pm 0.5$ & $2.4 \pm 0.2$ & $2.3 \pm 0.2$ & $2.4 \pm 0.2$ & $2.6 \pm 0.4$ & $1.9 \pm 0.1$ \\
\hline R. facians & $1 \pm 0.3$ & $3.8 \pm 0.2$ & $4.4 \pm 0.2$ & $4.4 \pm 0.2$ & $4 \pm 0.2$ & $1 \pm 0.2$ & $4.7 \pm 0.2$ & $4.5 \pm 0.2$ & $3.9 \pm 0.2$ & $5.0 \pm 0.3$ & $3.8 \pm 0.2$ & $3.6 \pm 0.2$ & $3.8 \pm 0.1$ & $4.4 \pm 0.3$ & $2.7 \pm 0.2$ \\
\hline S. scabies & $19.2 \pm 0.5$ & $12.3 \pm 0.3$ & $15.1 \pm 0.4$ & $14.8 \pm 0.3$ & $.7 \pm 0.3$ & $7.8 \pm 0.3$ & $16.0 \pm 0.4$ & $15.5 \pm 0.4$ & $12.9 \pm 0.3$ & $20.3 \pm 0.6$ & $13.0 \pm 0.3$ & $13.1 \pm 0.2$ & $13.4 \pm 0.3$ & $18.4 \pm 0.4$ & $10.3 \pm 0.3$ \\
\hline$X$. arboricola & $8.3 \pm 1.4$ & $5.6 \pm 0.8$ & $6.9 \pm 1.1$ & $6.8 \pm 1.0$ & $3 \pm 0.5$ & $3.9 \pm 0.4$ & $7.3 \pm 1.1$ & $7.0 \pm 1.1$ & $5.7 \pm 0.8$ & $8.9 \pm 1.5$ & $5.9 \pm 0.8$ & $5.7 \pm 0.9$ & $5.9 \pm 0.9$ & $8.3 \pm 1.4$ & $4.3 \pm 0.6$ \\
\hline$X$. fragariae & $2.3 \pm 0.2$ & $2.0 \pm 0.2$ & $2.2 \pm 0.1$ & $2.2 \pm 0.2$ & $2.0 \pm 0.2$ & $1.9 \pm 0.1$ & $2.2 \pm 0.2$ & $2.2 \pm 0.2$ & $2.0 \pm 0.1$ & $2.1 \pm 0.1$ & $2.1 \pm 0.1$ & $1.9 \pm 0.2$ & $2.0 \pm 0.2$ & $2.0 \pm 0.2$ & $1.7 \pm 0.1$ \\
\hline X. oryzae & $0 \pm 0.2$ & $1.6 \pm 0.1$ & $1.8 \pm 0.2$ & $1.9 \pm 0.2$ & $.7 \pm 0.2$ & $.6 \pm 0.1$ & $2.0 \pm 0.1$ & $1.9 \pm 0.2$ & $1.7 \pm 0.1$ & $1.9 \pm 0.2$ & $1.7 \pm 0.2$ & $1.6 \pm 0.1$ & $1.6 \pm 0.1$ & $1.7 \pm 0.2$ & $1.5 \pm 0.1$ \\
\hline NBY contr & \pm 0.1 & $21+01$ & $3+01$ & $3+01$ & $3 \pm 0.1$ & +01 & $2.3 \pm 0.1$ & $2.3 \pm 0.1$ & $2.2 \pm 0.1$ & $2.0 \pm 0.1$ & $2.3 \pm 0.1$ & $2.1 \pm 0.1$ & $2.2 \pm 0.1$ & $2.0 \pm 0.1$ & $1.8 \pm 0.1$ \\
\hline \multicolumn{16}{|l|}{ Fungi } \\
\hline A. mellea & \pm 0.2 & $8 \pm 0.2$ & $1.9 \pm 0.2$ & $2.0 \pm 0.2$ & $2.0 \pm 0.2$ & $2.0 \pm 0.2$ & $2.0 \pm 0.2$ & $2.0 \pm 0.2$ & $1.8 \pm 0.2$ & $1.8 \pm 0.3$ & $1.9 \pm 0.2$ & $1.8 \pm$ & $1.8 \pm 0.2$ & $1.8 \pm$ & $1.9 \pm 0.2$ \\
\hline A. tabesce & & $2.0 \pm 0.1$ & $2.2 \pm 0.1$ & $23+1$ & $2.2 \pm 0.1$ & & $2.3 \pm 0.1$ & $2.3 \pm 0.1$ & & & & & & & \\
\hline C. fagacearum & $2.1 \pm 0.1$ & $1.7 \pm 0.1$ & $1.9 \pm 0.1$ & $2.0 \pm 0.1$ & $1.8 \pm 0.1$ & $1.8 \pm 0.1$ & $2.0 \pm 0.1$ & $2.0 \pm 0.1$ & $1.7 \pm 0.1$ & $2.0 \pm 0.1$ & $1.8 \pm 0.1$ & $1.8 \pm 0.1$ & $1.8 \pm 0.1$ & $2.0 \pm 0.1$ & $1.8 \pm 0.1$ \\
\hline C. parasitica & $2.0 \pm 0.1$ & $1.7 \pm 0.1$ & $1.9 \pm 0.1$ & $1.9 \pm 0.1$ & $1.8 \pm 0.1$ & $1.8 \pm 0.1$ & $2.0 \pm 0.1$ & $2.0 \pm 0.1$ & $1.7 \pm 0.1$ & $1.9 \pm 0.1$ & $1.8 \pm 0.1$ & $1.7 \pm 0.1$ & $1.7 \pm 0.1$ & $2.0 \pm 0.2$ & $1.8 \pm 0.1$ \\
\hline D. quercina & \pm 0.1 & $2.0 \pm 0.1$ & $2.2 \pm 0.1$ & $2.3 \pm 0.1$ & $2.1 \pm 0.1$ & $2.1 \pm 0.1$ & $2.3 \pm 0.1$ & $2.3 \pm 0.1$ & $2.0 \pm 0.1$ & $2.2 \pm 0.1$ & $2.1 \pm 0.1$ & $2.0 \pm 0.1$ & $2.0 \pm 0.1$ & $2.2 \pm 0.1$ & $2.1 \pm 0.1$ \\
\hline G. lucidum & $1.7 \pm 0.1$ & $1.4 \pm 0.1$ & $1.6 \pm 0.1$ & $1.7 \pm 0.1$ & $1.5 \pm 0.1$ & $1.6 \pm 0.1$ & $1.7 \pm 0.1$ & $1.7 \pm 0.1$ & $1.5 \pm 0.1$ & $1.5 \pm 0.1$ & $1.5 \pm 0.1$ & $1.5 \pm 0.1$ & $1.5 \pm 0.1$ & $1.6 \pm 0.1$ & $1.5 \pm 0.1$ \\
\hline H. erinaceus & $2.1 \pm 0.1$ & $1.8 \pm 0.1$ & $2.0 \pm 0.1$ & $2.1 \pm 0.1$ & $1.9 \pm 0.1$ & $2.0 \pm 0.1$ & $2.1 \pm 0.1$ & $2.1 \pm 0.1$ & $1.9 \pm 0.1$ & $1.9 \pm 0.1$ & $1.9 \pm 0.1$ & $1.8 \pm 0.1$ & $1.8 \pm 0.1$ & $2.0 \pm 0.1$ & $1.9 \pm 0.1$ \\
\hline I. dryadeus & $2.0 \pm 0.1$ & $1.7 \pm 0.1$ & $1.9 \pm 0.1$ & $2.0 \pm 0.1$ & $1.8 \pm 0.1$ & $1.8 \pm 0.1$ & $2.0 \pm 0.1$ & $1.9 \pm 0.1$ & $1.7 \pm 0.1$ & $1.8 \pm 0.2$ & $1.8 \pm 0.1$ & $1.7 \pm 0.1$ & $1.7 \pm 0.1$ & $1.9 \pm 0.2$ & $1.8 \pm 0.1$ \\
\hline L. sulphureus & $2.3 \pm 0.1$ & $1.9 \pm 0.1$ & $2.2 \pm 0.1$ & $2.2 \pm 0.1$ & $2.0 \pm 0.1$ & $2.1 \pm 0.1$ & $2.2 \pm 0.1$ & $2.2 \pm 0.1$ & $2.0 \pm 0.1$ & $2.2 \pm 0.1$ & $2.1 \pm 0.1$ & $2.0 \pm 0.1$ & $2.0 \pm 0.1$ & $2.2 \pm 0.1$ & $2.0 \pm 0.1$ \\
\hline O. piceae & $1.9 \pm 0.2$ & $1.6 \pm 0.2$ & $1.8 \pm 0.2$ & $1.9 \pm 0.2$ & $1.7 \pm 0.1$ & $1.7 \pm 0.2$ & $1.8 \pm 0.2$ & $1.8 \pm 0.2$ & $1.6 \pm 0.2$ & $1.7 \pm 0.3$ & $1.7 \pm 0.2$ & $1.6 \pm 0.2$ & $1.6 \pm 0.2$ & $1.8 \pm 0.3$ & $1.7 \pm 0.2$ \\
\hline O. pluriannulatum & $1.9 \pm 0.2$ & $1.7 \pm 0.2$ & $1.9 \pm 0.2$ & $1.9 \pm 0.2$ & $1.7 \pm 0.1$ & $1.8 \pm 0.1$ & $1.9 \pm 0.2$ & $1.9 \pm 0.2$ & $1.7 \pm 0.2$ & $1.8 \pm 0.3$ & $1.8 \pm 0.2$ & $1.7 \pm 0.2$ & $1.7 \pm 0.2$ & $1.9 \pm 0.3$ & $1.8 \pm 0.2$ \\
\hline P. ostreatus & $2.7 \pm 0.1$ & $2.4 \pm 0.1$ & $2.6 \pm 0.1$ & $2.7 \pm 0.1$ & $2.5 \pm 0.1$ & $2.6 \pm 0.1$ & $2.7 \pm 0.2$ & $2.7 \pm 0.1$ & $2.4 \pm 0.1$ & $2.6 \pm 0.2$ & $2.5 \pm 0.1$ & $2.4 \pm 0.1$ & $2.4 \pm 0.1$ & $2.7 \pm 0.2$ & $2.4 \pm 0.1$ \\
\hline P. graminicola & $2.3 \pm 0.2$ & $1.9 \pm 0.2$ & $2.1 \pm 0.2$ & $2.1 \pm 0.2$ & $1.9 \pm 0.2$ & $1.9 \pm 0.2$ & $2.2 \pm 0.2$ & $2.2 \pm 0.2$ & $1.9 \pm 0.2$ & $2.1 \pm 0.3$ & $2.0 \pm 0.2$ & $1.9 \pm 0.2$ & $1.9 \pm 0.2$ & $2.2 \pm 0.2$ & $2.0 \pm 0.2$ \\
\hline P. oligandrum & $2.3 \pm 0.1$ & $1.9 \pm 0.1$ & $2.2 \pm 0.1$ & $2.2 \pm 0.1$ & $2.0 \pm 0.1$ & $2.0 \pm 0.1$ & $2.2 \pm 0.2$ & $2.2 \pm 0.2$ & $2.0 \pm 0.1$ & $2.2 \pm 0.2$ & $2.0 \pm 0.1$ & $2.0 \pm 0.2$ & $2.0 \pm 0.2$ & $2.2 \pm 0.2$ & $2.0 \pm 0.2$ \\
\hline P. ultimum & $1.6 \pm 0.1$ & $1.3 \pm 0.1$ & $1.5 \pm 0.1$ & $1.6 \pm 0.1$ & $1.4 \pm 0.1$ & $1.4 \pm 0.1$ & $1.6 \pm 0.2$ & $1.5 \pm 0.1$ & $1.4 \pm 0.1$ & $1.5 \pm 0.2$ & $1.4 \pm 0.1$ & $1.4 \pm 0.1$ & $1.4 \pm 0.1$ & $1.6 \pm 0.2$ & $1.4 \pm 0.1$ \\
\hline S. commune & $2.7 \pm 0.1$ & $2.3 \pm 0.1$ & $2.5 \pm 0.1$ & $2.6 \pm 0.1$ & $2.3 \pm 0.1$ & $2.4 \pm 0.1$ & $2.6 \pm 0.1$ & $2.6 \pm 0.1$ & $2.3 \pm 0.1$ & $2.6 \pm 0.1$ & $2.4 \pm 0.1$ & $2.4 \pm 0.1$ & $2.4 \pm 0.1$ & $2.6 \pm 0.1$ & $2.4 \pm 0.1$ \\
\hline T. versicolor & $2.6 \pm 0.1$ & $2.2 \pm 0.1$ & $2.5 \pm 0.1$ & $2.6 \pm 0.1$ & $2.4 \pm 0.1$ & $2.4 \pm 0.1$ & $2.6 \pm 0.1$ & $2.5 \pm 0.1$ & $2.3 \pm 0.1$ & $2.4 \pm 0.2$ & $2.4 \pm 0.1$ & $2.2 \pm 0.1$ & $2.3 \pm 0.1$ & $2.5 \pm 0.2$ & $2.3 \pm 0.1$ \\
\hline MA control & $2.1 \pm 0.1$ & $1.9 \pm 0.1$ & $2.1 \pm 0.1$ & $2.2 \pm 0.1$ & $2.0 \pm 0.1$ & $2.0 \pm 0.1$ & $2.1 \pm 0.1$ & $2.1 \pm 0.1$ & $1.9 \pm 0.1$ & $2.0 \pm 0.1$ & $2.0 \pm 0.1$ & $1.9 \pm 0.1$ & $1.9 \pm 0.1$ & $2.1 \pm 0.1$ & $1.9 \pm 0.1$ \\
\hline
\end{tabular}

a NBY = nutrient-broth yeast extract agar, and MA = malt agar.

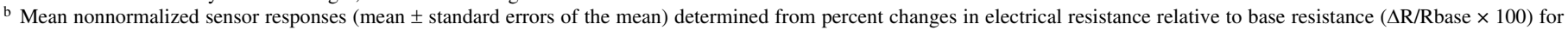
each individual sensor with 10 replicate runs per strain of each microbial species, and multiple strains ( 2 to 10 per species) with few exceptions.

the array, were generally those that produced the most noticeably pungent, volatile aromas in culture. The headspace volatiles of filamentous bacterium $S$. scabies caused consistently very high sensor responses across the sensor array, indicative of compounds with high affinity for all of the different types of conductive polymer coatings on sensors. Headspace volatiles from fungi rarely produced nonnormalized sensor-response intensities of $>3 \%$ using the general-use A32S sensor array. Nevertheless, sufficient differences in individual sensor responses were produced and EASPs were sufficiently unique for different fungi to allow discrimination between fungal species. Individual nonnormalized sensor intensity responses were rarely $<1 \%$ for fungi, but intensity responses of $<1 \%$ were observed with some bacteria. Certain anaerobic bacteria, such as those causing wetwood in hardwoods, often released copious quantities of carboxylic acids into the sampled headspace, producing negative responses for sensors that were sensitive to polar compounds.

The EASPs of microbes in graphic formats were useful visual references for comparing sample unknowns to known EASPs during methods development, reference database construction, and comparisons of closely related microbes. For example, the EASPs of two closely related bacteria, $R$. rhizogenes (=Agrobacterium rhizogenes) and $R$. radiobacter (=Agrobacterium tumefaciens), were compared using two types of CPA signature-differentiation display modes - superimpose (simultaneous) mode and difference mode. The EASPs were compared separately using actual (non- normalized) and normalized data (Fig. 2). Histogram comparisons using normalized EASPs of these bacteria indicated considerable differences in sensor responses to their headspace volatiles in superimpose mode (Fig. 2A). Relative differences in sensor responses to volatiles of $R$. radiobacter compared with $R$. rhizogenes were indicated in the difference display mode (Fig. 2B). Comparisons using nonnormalized data as line graphs provided views of actual raw-data differences in sensor responses using superimpose mode (Fig. 2C) and in difference mode (Fig. 2D). With both normalized and nonnormalized data, difference mode indicated differences in sensor responses in the EASPs of one bacterium relative to those of the other bacterium. Thus, it mattered which was the EASP of the first bacterium $\left(\mathrm{R}_{1}\right)$ that was used as the reference pattern to which the EASP of the second bacterium $\left(\mathrm{R}_{2}\right)$ was compared because corresponding sensor responses were reversed along the $x$ axis at approximately the zeroresponse line for normalized histogram data, but no change occurred for nonnormalized data. This result occurred because the analysis software selects the curve with the highest values as the reference curve $\left(R_{1}\right)$. The curve with the smaller values $\left(R_{2}\right)$ becomes a flat reference line following subtraction of nonnormalized data (Fig. 2D).

Determinations of infection status in living host tissues. Headspace volatiles released from tree cores that were extracted from the outer sapwood of live oaks were used to test the ability of CPA to diagnose oak wilt in living trees at various stages of 


\begin{tabular}{|c|c|c|c|c|c|c|c|c|c|c|c|c|c|c|c|c|}
\hline \multicolumn{17}{|c|}{ Sensor number ${ }^{b}$} \\
\hline 16 & 17 & 18 & 19 & 20 & 21 & 22 & 23 & 24 & 25 & 26 & 27 & 28 & 29 & 30 & 31 & 32 \\
\hline $2.2 \pm 0.1$ & \pm 0.1 & \pm 0.1 & \pm 0.1 & \pm 0.1 & 0.1 & \pm 0.1 & \pm 0.1 & 1 & $=0.1$ & \pm 0.1 & $5 \pm 0.1$ & $5 \pm 0.1$ & $2.2 \pm 0.1$ & $2.9 \pm 0.1$ & $4 \pm 0.1$ & $.3 \pm 0.1$ \\
\hline $2.2 \pm 0.2$ & $6 \pm 0.4$ & $1 \pm 0.2$ & $2.6 \pm 0.4$ & $2.7 \pm 0.4$ & $1.8 \pm 0.2$ & $1.6 \pm 0.1$ & $3 \pm 0.1$ & $3 \pm 0.1$ & $2.0 \pm 0.2$ & $2.9 \pm 0.4$ & $2.4 \pm 0.3$ & $2.4 \pm 0.3$ & $2.2 \pm 0.2$ & $2.7 \pm 0.4$ & $2.3 \pm 0.3$ & $2.2 \pm 0.3$ \\
\hline $4.3 \pm 0.2$ & $5.8 \pm 0.3$ & $2 \pm 0.2$ & $6 \pm 0.1$ & $6.5 \pm 0.2$ & $3.5 \pm 0.1$ & $2.8 \pm 0.1$ & $7 \pm 0.1$ & $2 \pm 0.2$ & $.0 \pm 0.2$ & $6.9 \pm 0.3$ & $5.3 \pm 0.3$ & $5.3 \pm 0.3$ & $4.3 \pm 0.2$ & $6.0 \pm 0.3$ & $.2 \pm 0.2$ & \\
\hline $1.7 \pm 0.1$ & $2.0 \pm 0.1$ & $7 \pm 0.1$ & $1.7 \pm 0.1$ & $1.9 \pm 0.1$ & $1.5 \pm 0.1$ & $1.4 \pm 0.0$ & $.3 \pm 0.0$ & $3 \pm 0.1$ & $1.6 \pm 0.1$ & $2.1 \pm 0.1$ & $1.8 \pm 0.1$ & $1.8 \pm 0.1$ & $1.7 \pm 0.1$ & $2.1 \pm 0.1$ & $1.6 \pm 0.1$ & $1.7 \pm 0.1$ \\
\hline $0.9 \pm 0.1$ & $1.1 \pm 0.1$ & $0.9 \pm 0.1$ & $1.1 \pm 0.1$ & $1.0 \pm 0.1$ & $0.7 \pm 0.0$ & & & & & & & & & & & \\
\hline $6.0 \pm 0.1$ & $7.6 \pm 0.1$ & $5.4 \pm 0.1$ & $8.7 \pm 0.2$ & $8.6 \pm 0.2$ & $4.9 \pm 0.1$ & $3.6 \pm 0.1$ & $3 \pm 0.1$ & $6 \pm 0.0$ & $6 \pm 0.2$ & $.2 \pm 0.2$ & $7.2 \pm 0.1$ & $7.3 \pm 0.1$ & $6.0 \pm 0.0$ & $8.1 \pm 0.1$ & $7.1 \pm 0.1$ & $6.3 \pm 0.1$ \\
\hline $2.4 \pm 0.2$ & $2.9 \pm 0.3$ & $2.3 \pm 0.2$ & $2.7 \pm 0.3$ & $2.9 \pm 0.3$ & $2.0 \pm 0.1$ & $1.8 \pm 0.1$ & $1.5 \pm 0.1$ & $.4 \pm 0.0$ & $2.2 \pm 0.2$ & $3.1 \pm 0.3$ & $2.6 \pm 0.2$ & $2.6 \pm 0.2$ & $2.4 \pm$ & $3.0 \pm$ & $2.4 \pm$ & $2.4 \pm$ \\
\hline $5.5 \pm 0.2$ & $7.5 \pm 0.3$ & $1 \pm 0.2$ & $9.2 \pm 0.4$ & $8.5 \pm 0.3$ & $4.4 \pm 0.2$ & $3.2 \pm 0.2$ & $1.9 \pm 0.2$ & $2 \pm 0.1$ & $5.2 \pm 0.3$ & $8.8 \pm 0.4$ & $6.8 \pm 0.3$ & $6.9 \pm 0.3$ & $5.5 \pm 0.2$ & $7.6 \pm 0.3$ & $6.7 \pm$ & $5.8 \pm$ \\
\hline $1.8 \pm 0.1$ & $1.7 \pm 0.1$ & $1.7 \pm 0.1$ & $1.5 \pm 0.1$ & $1.8 \pm 0.1$ & $1.8 \pm 0.1$ & $1.7 \pm 0.1$ & $1.8 \pm 0.1$ & $1.9 \pm 0.1$ & $1.7 \pm 0.1$ & $2.0 \pm 0.1$ & $1.8 \pm 0.1$ & $1.9 \pm$ & $2.0 \pm 0.1$ & $2.1 \pm$ & $1.6 \pm$ & $2.1=$ \\
\hline $3.6 \pm 0.2$ & $4.8 \pm 0.2$ & $7 \pm 0.2$ & $5.2 \pm 0.1$ & $5.2 \pm 0.2$ & $2.9 \pm$ & & \pm 0.1 & & & $5.6 \pm$ & & $4.3 \pm$ & & & & \\
\hline $5.8 \pm 0.2$ & $7.8 \pm 0.2$ & $5.5 \pm 0.1$ & $9.2 \pm 0.1$ & $8.6 \pm 0.3$ & $4.7 \pm 0.0$ & $3.5 \pm 0.1$ & $2.3 \pm 0.2$ & $1.6 \pm 0.1$ & $5.4 \pm 0.2$ & $9.0 \pm 0.1$ & $7.1 \pm 0.1$ & $7.1 \pm 0.1$ & $5.8 \pm 0.1$ & $7.8 \pm 0.0$ & $6.9 \pm$ & $6.1 \pm$ \\
\hline $2.1 \pm 0.1$ & $2.3 \pm 0.2$ & $2.0 \pm 0.1$ & $1.9 \pm 0.2$ & $2.2 \pm 0.2$ & $1.9 \pm 0.1$ & $1.8 \pm 0.0$ & \pm 0.1 & \pm 0.1 & $1.9 \pm 0.1$ & $2.5 \pm$ & $2.2 \pm 0.1$ & $2.2 \pm$ & $2.2 \pm$ & $2.5 \pm$ & & \\
\hline $3.0 \pm 0.2$ & $3.4 \pm 0.2$ & $2.8 \pm 0.1$ & $3.1 \pm 0.2$ & $3.3 \pm 0.2$ & $2.5 \pm$ & $2.3 \pm 0.1$ & $2.0 \pm 0.1$ & $2.1 \pm$ & $2.6 \pm 0.2$ & $3.6 \pm 0.2$ & $3.0 \pm 0.2$ & $3.1 \pm$ & $2.9 \pm 0.2$ & $3.5 \pm 0.2$ & $2.9 \pm$ & $3.0 \pm$ \\
\hline $11.2 \pm 0.1$ & $12.6 \pm 0.4$ & $8.6 \pm 0.3$ & $16.0 \pm 0.4$ & $15.0 \pm 0.3$ & $9.3 \pm$ & & $3.7 \pm 0.2$ & $.5 \pm 0.2$ & $10.3 \pm 0.3$ & $15.6 \pm 0.4$ & $12.9 \pm 0.3$ & $13.4 \pm 0.3$ & $11.4 \pm$ & $14.1 \pm$ & 12.5 & \\
\hline $4.9 \pm 0.7$ & $5.6 \pm 0.8$ & $4.2 \pm 0.5$ & $6.5 \pm 1.1$ & $6.4 \pm 1.0$ & $4.1 \pm$ & $3.0 \pm 0.3$ & $2.3 \pm 0.2$ & $2.0 \pm 0.1$ & $4.4 \pm 0.6$ & $6.6 \pm 1.0$ & $5.5 \pm 0.8$ & $5.4 \pm 0.8$ & & & & \\
\hline & & & & & & & & & & & & & & & & \\
\hline $1.5 \pm 0.1$ & $1.4 \pm 0.1$ & $1.3 \pm 0.1$ & $1.3 \pm 0.1$ & $1.4 \pm 0.1$ & $1.4 \pm 0.1$ & $1.3 \pm 0.1$ & $1.4 \pm 0.1$ & $1.5 \pm 0.1$ & & $1.6 \pm 0.1$ & $1.5 \pm 0.1$ & $1.5 \pm 0.1$ & & $1.7 \pm 0.2$ & & $1.7 \pm 0.1$ \\
\hline $2.0 \pm 0.1$ & $2.0 \pm 0.1$ & & & & & & & & & & & & & & & \\
\hline & & & & & & & & & & & & & & & & \\
\hline $2.1 \pm 0.1$ & $2.0 \pm 0.1$ & $0 \pm$ & $9 \pm$ & 2 & $2.0 \pm$ & $2.0 \pm$ & .1 & 2 & $1.9=$ & $2.3=$ & 2.2 & 1 & & & 2.0 & \\
\hline $1.8 \pm 0.1$ & $1.7 \pm 0.1$ & $1.6 \pm 0.1$ & $1.8 \pm 0.1$ & $2.0 \pm 0.1$ & $1.7 \pm 0.1$ & $1.5 \pm 0.1$ & $1.6 \pm 0.1$ & $1.7 \pm 0.1$ & $1.6 \pm 0.1$ & $1.9 \pm 0.1$ & $1.8 \pm 0.1$ & $1.9 \pm$ & $2.0 \pm$ & $2.0 \pm$ & 1.6 & \\
\hline $1.8 \pm 0.1$ & $1.7 \pm 0.1$ & $1.6 \pm 0.1$ & $1.7 \pm 0.2$ & $1.9 \pm 0.1$ & $1.7 \pm 0.1$ & $1.5 \pm 0.1$ & $1.6 \pm 0.1$ & $1.6 \pm 0.1$ & $1.6 \pm 0.1$ & $1.9 \pm 0.1$ & $1.8 \pm 0.1$ & $1.9 \pm 0.1$ & $1.9 \pm 0.1$ & $2.0 \pm 0.1$ & $1.6 \pm$ & $1.9 \pm$ \\
\hline $2.1 \pm 0.1$ & $2.0 \pm 0.1$ & $1.9 \pm 0.0$ & $2.1 \pm 0.1$ & $2.2 \pm 0.1$ & $2.0 \pm 0.1$ & $1.8 \pm 0.0$ & $1.8 \pm 0.0$ & & $1.8 \pm 0.1$ & $2.2 \pm 0.1$ & $2.1 \pm 0.1$ & $2.1 \pm 0.1$ & & $2.3 \pm 0.1$ & $1.8 \pm$ & \\
\hline $1.5 \pm 0.1$ & $1.5 \pm 0.1$ & $4 \pm 0.1$ & $1.4 \pm 0.1$ & $1.6 \pm 0.1$ & $1.5 \pm 0.1$ & $1.3 \pm 0.1$ & $1.4 \pm 0.1$ & $1.4 \pm 0.1$ & $1.4 \pm 0.1$ & $1.7 \pm$ & $1.5 \pm 0.1$ & $1.6 \pm$ & $1.7 \pm$ & $1.8 \pm$ & $1.4=$ & $1.7 \pm 0.1$ \\
\hline $1.9 \pm 0.1$ & $1.8 \pm 0.1$ & $1.7 \pm 0.1$ & $1.8 \pm 0.1$ & $2.1 \pm 0.1$ & $1.8 \pm 0.1$ & $1.6 \pm 0.1$ & $1.7 \pm 0.1$ & $1.8 \pm 0.1$ & $1.7 \pm 0.1$ & $2.0 \pm 0.1$ & $1.9 \pm 0.1$ & $1.9 \pm 0.1$ & & $2.1 \pm 0.1$ & $1.7 \pm$ & \\
\hline $1.8 \pm 0.1$ & $1.7 \pm 0.1$ & $1.6 \pm 0.1$ & $1.7 \pm 0.2$ & $1.9 \pm 0.2$ & $1.7 \pm 0.1$ & $1.5 \pm 0.1$ & $1.6 \pm 0.1$ & $1.6 \pm 0.1$ & $1.6 \pm 0.1$ & $1.9 \pm 0.1$ & $1.8 \pm 0.1$ & $1.8 \pm 0.1$ & $1.9 \pm$ & $2.0 \pm 0.1$ & $1.6=$ & $1.9 \pm 0.1$ \\
\hline $2.1 \pm 0.1$ & $2.0 \pm 0.1$ & $1.8 \pm 0.1$ & $2.0 \pm 0.1$ & & $1.9 \pm 0.1$ & & $1.8 \pm 0.1$ & $1.9 \pm 0.1$ & & $2.2 \pm 0.1$ & $2.1 \pm 0.1$ & $2.2 \pm 0.1$ & & $2.3 \pm 0.1$ & $1.8 \pm$ & \\
\hline $1.7 \pm 0.2$ & $1.6 \pm 0.2$ & $1.5 \pm 0.1$ & $1.6 \pm 0.3$ & $1.8 \pm 0.2$ & $1.6 \pm 0.1$ & $1.5 \pm 0.1$ & $1.5 \pm 0.1$ & $1.6 \pm 0.1$ & $1.5 \pm 0.2$ & $1.8 \pm 0.2$ & $1.7 \pm 0.2$ & $1.7 \pm 0.2$ & $1.9 \pm 0.2$ & $1.9 \pm 0.2$ & $1.5 \pm 0.1$ & $1.9 \pm 0.2$ \\
\hline $1.8 \pm 0.2$ & $1.7 \pm 0.2$ & $1.6 \pm 0.1$ & $1.7 \pm 0.3$ & $1.9 \pm 0.3$ & $1.7 \pm 0.2$ & $1.5 \pm 0.1$ & $1.6 \pm 0.1$ & $1.6 \pm 0.1$ & $1.6 \pm 0.2$ & $1.9 \pm 0.2$ & $1.8 \pm 0.2$ & $1.8 \pm 0.2$ & $1.9 \pm 0.2$ & $2.0 \pm 0.2$ & $1.6 \pm 0.1$ & $1.9 \pm 0.2$ \\
\hline $2.5 \pm 0.1$ & $2.4 \pm 0.1$ & $2.3 \pm 0.1$ & & & $2.3 \pm 0.1$ & & $2.3 \pm 0.1$ & & & $2.6 \pm 0.1$ & $2.5 \pm 0.1$ & & & & & $2.7 \pm 0.1$ \\
\hline $2.0 \pm 0.2$ & $1.9 \pm 0.2$ & $1.7 \pm 0.1$ & $2.0 \pm 0.3$ & $2.1 \pm 0.2$ & $1.9 \pm 0.2$ & $1.7 \pm 0.1$ & $1.7 \pm 0.1$ & $1.8 \pm 0.1$ & $1.7 \pm 0.2$ & $2.1 \pm 0.2$ & $2.0 \pm 0.2$ & $2.1 \pm 0.2$ & $2.2 \pm 0.2$ & $2.2 \pm 0.2$ & $1.8 \pm 0.1$ & $2.1 \pm 0.2$ \\
\hline $2.1 \pm 0.2$ & $2.0 \pm 0.1$ & $1.8 \pm 0.1$ & $2.0 \pm 0.3$ & & $1.9 \pm 0.1$ & & & & & $2.2 \pm 0.1$ & $2.0 \pm 0.1$ & & & & & $2.2 \pm 0.1$ \\
\hline $1.5 \pm 0.1$ & $1.4 \pm 0.1$ & $1.3 \pm 0.1$ & $1.5 \pm 0.2$ & $1.6 \pm 0.1$ & $1.4 \pm 0.1$ & $1.2 \pm 0.1$ & $1.3 \pm 0.1$ & $1.3 \pm 0.1$ & $1.3 \pm 0.1$ & $1.6 \pm 0.1$ & $1.5 \pm 0.1$ & $1.5 \pm 0.1$ & $1.6 \pm 0.1$ & $1.7 \pm 0.1$ & $1.3 \pm 0.1$ & $1.6 \pm 0.1$ \\
\hline $2.4 \pm 0.1$ & $2.3 \pm 0.1$ & $2.1 \pm 0.1$ & $2.4 \pm 0.1$ & $2.6 \pm 0.1$ & $2.3 \pm 0.1$ & $2.1 \pm 0.1$ & $2.1 \pm 0.1$ & $2.2 \pm 0.1$ & $2.1 \pm 0.1$ & $2.6 \pm 0.1$ & $2.4 \pm 0.1$ & $2.5 \pm 0.1$ & $2.6 \pm 0.1$ & $2.7 \pm 0.1$ & $2.2 \pm 0.1$ & $2.6 \pm 0.1$ \\
\hline $2.3 \pm 0.1$ & $2.2 \pm 0.1$ & $2.1 \pm 0.1$ & $2.3 \pm 0.2$ & $2.6 \pm 0.2$ & $2.2 \pm 0.1$ & $2.1 \pm 0.1$ & $2.2 \pm 0.1$ & $2.3 \pm 0.1$ & $2.0 \pm 0.1$ & $2.5 \pm 0.1$ & $2.3 \pm 0.1$ & $2.3 \pm 0.1$ & $2.5 \pm 0.1$ & $2.6 \pm 0.1$ & $2.1 \pm 0.1$ & $2.5 \pm 0.1$ \\
\hline $2.0 \pm 0.1$ & $1.9 \pm 0.1$ & $1.8 \pm 0.1$ & $1.9 \pm 0.1$ & $2.1 \pm 0.1$ & $1.8 \pm 0.1$ & $1.7 \pm 0.1$ & $1.7 \pm 0.1$ & $1.8 \pm 0.1$ & $1.7 \pm 0.1$ & $2.1 \pm 0.1$ & $1.9 \pm 0.1$ & $1.9 \pm 0.1$ & $2.1 \pm 0.1$ & $2.2 \pm 0.1$ & $1.7 \pm 0.1$ & $2.1 \pm 0.1$ \\
\hline
\end{tabular}

infection. Mean global class distributions provided indications of the percentages of aroma elements in the electronic signature that matched those of three aroma global class categories (healthy, infected, and unknown) in the reference library. Global class membership values of $>90 \%$ were strong indicators of sample identity, in this case, oak wilt infection status. Aroma profiles of sapwood cores from healthy and inoculated controls had very high levels of global class membership in the infection categories healthy and infected, respectively, consistent with their source and infection status (Table 4). All aroma elements of EASPs from healthy nonhost control cores were predominantly unknown, with only minor membership distributions among healthy and infected global classes. Healthy (uninfected) live oak cores produced results consistent with healthy controls, having almost all aroma elements identified in the healthy global class category. Sapwood cores from weakly symptomatic, oak wilt-infected live oaks had high global class membership (almost 80\%) in the infected category, with a significant portion remaining in the healthy category. Thus, a mean global class membership value of $<90 \%$ indicated that core samples from weakly symptomatic trees were not always reliably diagnosed. Cores from strongly symptomatic infected trees were identified in the infected category, but had minor elements in the unknown and healthy categories.

Reliability of sample identifications and disease diagnoses. The efficacy of CPA in providing accurate microbial identifications in vitro, identifications of host tissues in vivo, and diagnoses of specific diseases were tested using headspace volatiles from cultures and excised sapwood cores from healthy and diseased host plants, respectively. All strains of pathogenic and wooddecay fungi were identified correctly at high levels of confidence (Table 5). The only exception was Ganoderma lucidum, for which only $89 \%$ of strains were correctly identified. The remaining strains of G. lucidum could not be identified. Mean global class distributions indicated $>95 \%$ correct identifications for microbes in the proper category. Similar results were obtained for the identifications of plant hosts for most plant species tested. Identifications of Diospyros virginiana (American persimmon) and Prosopis glandulosa (mesquite) sapwood cores using CPA were somewhat less reliable at 90 and $80 \%$, respectively. The remaining specimens from these two species could not be identified (Table 5). Global class distributions in the correct categories also were very high among the host species tested.

The results for tests of infection status or diagnosis of specific diseases in host tissues were more variable than for analysis of volatiles from microbes or host tissue samples individually (Table $5)$. The reliability of diagnoses of oak wilt infections in live oaks was dependent upon infection status and levels of infection. Identification of infection status in healthy controls and uninfected samples was most reliable (100\% identified correctly) with samples containing only host volatiles to affect discrimination. Correct identification of inoculated control samples was next in reliability (97\%). In this case, host tissues initially were overwhelmed by inoculum and subsequent colonization by the oak wilt pathogen, $C$. fagacearum. Infected cores from strongly symptomatic plants also were detected reliably in $93 \%$ of specimens tested. However, only $\approx 80 \%$ of core samples from weakly 
symptomatic plants were identified as infected. None of the analyzed samples for which an identical global class existed in the reference library were incorrectly or ambiguously identified. The identity of all such samples that could not be determined was classified as unidentified (Table 5). Most healthy control samples from nonhost plants were classified as indeterminate or not identified. Approximately $4 \%$ of samples each were either misidentified as infected live oak or ambiguously identified. Ambiguous identifications indicate that separate samples of the same type were determined to have majority ownership in different global classes. Nonhost samples that lacked representative databases in the reference library were the only sample type that had determinations in these categories.

Tests of CPA reliability for the diagnosis of bacterial wetwood in Populus deltoides (cottonwood) using volatiles from sapwood cores provided more discrete results. Freshly excised sapwood core samples collected from trees in cottonwood plantations and at the saw mill were either strongly symptomatic (visibly dark, wet, and with ring shakes in annual rings) or healthy (white, dry, and structurally intact without ring shakes). However, it was harder to distinguish symptoms and visually discriminate between healthy and infected cores once they had dried after excision. The infected cores tended to lose the dark pigmentation and drying tended to strengthen structural rigidity. Both healthy and infected sapwood cores from cottonwood were identified with high levels of confidence based on differences in EASPs. The mean global class ownership of healthy and infected samples was very high in corresponding categories of proper identity.
Mean global class memberships of all microbial species, host tissues, and disease diagnoses were very high in the correct category of identification. Most samples that were not correctly identified could not be categorized to an identifiable global aroma class. No samples were identified incorrectly or ambiguously for which an aroma class category existed in the reference library.

TABLE 4. Mean global class membership of validated aroma profiles for sapwood cores of live oak trees in various categories of oak wilt infection, and of nonhost hardwood species

\begin{tabular}{lcccc}
\hline & & \multicolumn{3}{c}{ Mean global class distribution $(\%)^{\mathrm{b}}$} \\
\cline { 3 - 5 } Infection status of sample $^{\mathrm{a}}$ & $n$ & Healthy & Infected & Unknown \\
\hline Healthy (uninfected) & 15 & 99.9 & 0.0 & 0.1 \\
Weakly symptomatic infected & 15 & 19.5 & 79.8 & 0.7 \\
Strongly symptomatic infected & 15 & 1.8 & 93.8 & 4.4 \\
Healthy control & 39 & 99.9 & 0.0 & 0.1 \\
Inoculated control & 30 & 0.0 & 99.6 & 0.4 \\
Healthy nonhost control & 24 & 1.4 & 1.3 & 97.3 \\
\hline
\end{tabular}

a All samples were sapwood cores of Plateau live oak, Quercus fusiformis, except nonhost samples which were sapwood cores of various hardwood species immune to C. fagacearum infection.

${ }^{\mathrm{b}}$ Global classes distributions indicate the percentage of aroma elements in the electronic signature that match elements found in the electronic signatures of known healthy and infected samples in the reference library. Distribution values of $>90 \%$ are strong indicators of sample identity (infection status). Unknown designations indicate the percentage of elements in the sample that could not be identified or matched to any global classes found among databases included in the reference library used for sample determinations.

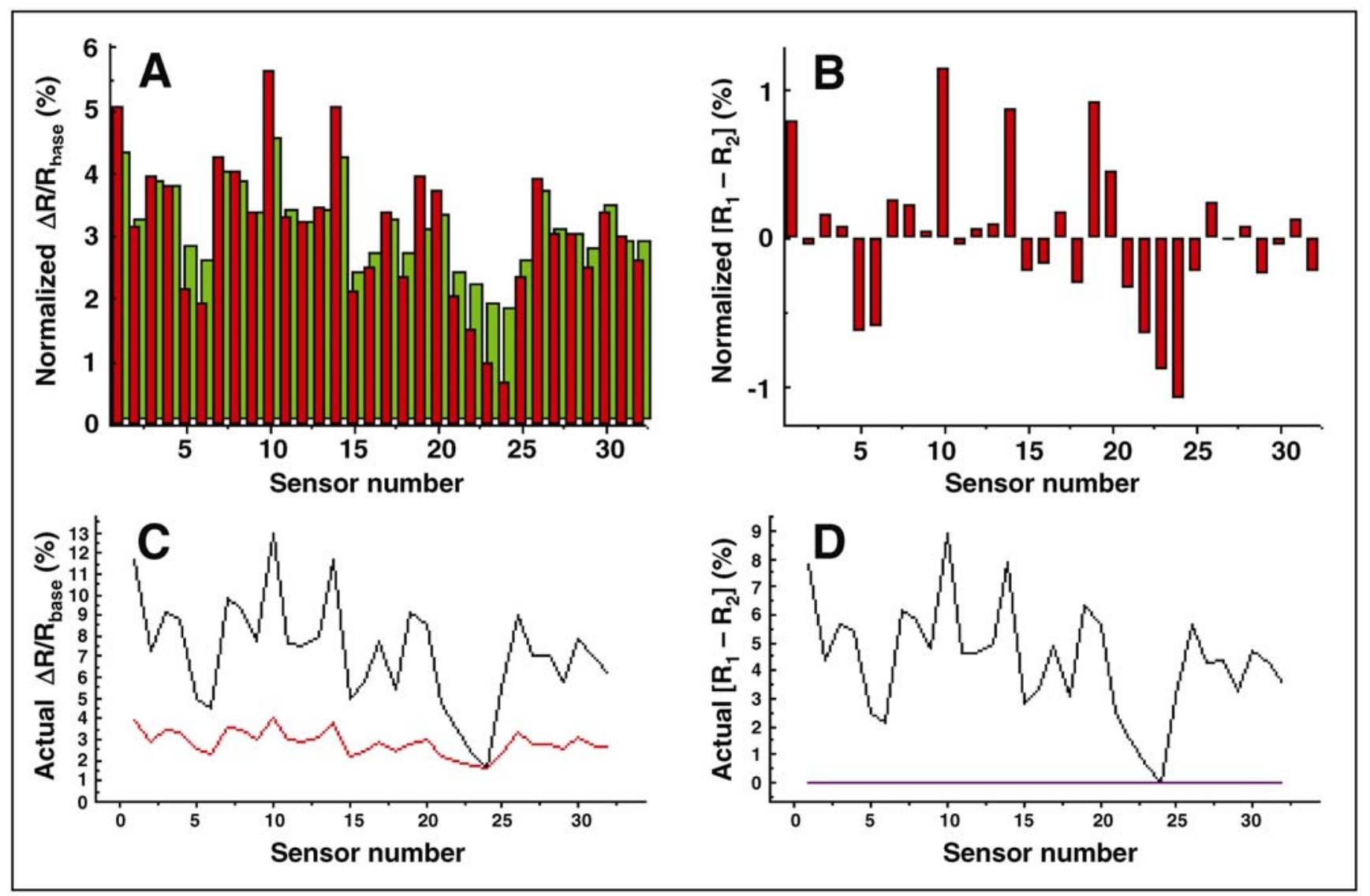

Fig. 2. Comparisons of actual and normalized intensity responses $\left(\% \Delta \mathrm{R} / \mathrm{R}_{\text {base }}\right)$ of the Aromascan $\mathrm{A} 32 \mathrm{~S}$ sensor array to headspace volatiles produced by $R h i$ zobium rhizogenes and $R$. radiobacter on nutrient-broth yeast extract agar using two types of conductive polymer analysis signature-differentiation modes. Histogram comparisons of the normalized electronic aroma signature pattern of $R$. radiobacter (green bars) compared with (relative to) the $R$. rhizogenes (red bars) reference pattern in A, superimpose mode, and in $\mathbf{B}$, difference mode. Graphed comparisons of actual (non-normalized) electronic aroma signature pattern of $R$. radiobacter (bottom orange or purple line) compared with $R$. rhizogenes (top dark gray line) in $\mathbf{C}$, superimpose mode, and in $\mathbf{D}$, difference modes. In the normalized and actual difference-mode comparisons, $\mathrm{R}_{1}=$ electronic aroma signature pattern of $R$. rhizogenes and $\mathrm{R}_{2}=$ electronic aroma signature pattern of R. radiobacter. 
PCA of related microbial species and for disease diagnoses. A critical comparison in all possible combinations of EASPs from headspace volatiles of closely related microbial species using three-dimensional PCA allowed quantitation of the relatedness of headspace mixtures produced and an indication of the relative taxonomic relatedness of compared species based on the volatile metabolites produced. These measurements were quantified with a quality factor $(\mathrm{QF})$ of significance value. The higher the QF significance, the greater the difference or less related the analytes compared. A quality factor value of 2.0 generally was considered to be a significant discrimination equivalent to approximately $P=$ 0.05 . Analysis of four species of phytopathogenic fungi in the Ceratocystis-Ophiostoma complex indicated high levels of significant difference between these species (Table 6). The EASP of C. fagacearum was significantly different from $C$. fimbriata, Ophiostoma piceae, and O. plurianulatum $(P<0.01)$. Even greater differences were determined between $C$. fimbriata and $O$. piceae
TABLE 6. Three-dimensional principal component analysis of headspace volatile metabolites released from cultures of Ceratocystis and Ophiostoma spp. growing on standardized malt-agar growth medium

\begin{tabular}{llc}
\hline Analyte 1 & Analyte 2 & QF significance $^{\mathrm{a}}$ \\
\hline Ceratocystis fagacearum & C. fimbriata & $11.83^{* *}$ \\
& Ophiostoma piceae & $11.93^{* *}$ \\
& O. pluriannulatum & $11.74^{* *}$ \\
C. fimbriata & O. piceae & $19.70^{* * *}$ \\
& O. pluriannulatum & $14.98^{* * *}$ \\
O. piceae & O. pluriannulatum & $36.57^{* * *}$ \\
\hline
\end{tabular}

${ }^{\mathrm{a}} \mathrm{QF}=$ quality factor. Discrimination between analytes (aroma classes) was significant at the following levels: ** and *** indicate $P<0.01$ and 0.001 , respectively. $\mathrm{A} \mathrm{QF}$ value of 2.0 indicates a significant discrimination at $\approx P=$ 0.05 . The percentages of the total variance, accounting for the variability explained by each orthogonal principal component (PC), are as follows: PC $1=$ $76.62 \%$, PC $2=8.93 \%$, and PC $3=4.27 \%$, representing the $x, y$, and $z$ axis of the aroma map, respectively.

TABLE 5. Reliability of microbial identifications in culture, host identifications from tree cores, and disease diagnoses as determined by conductive polymer analysis with the Aromascan A32S using recognition files constructed from sample-specific reference libraries

\begin{tabular}{|c|c|c|c|c|c|}
\hline Sample unknowns ${ }^{\mathrm{a}}$ & $n$ & $\begin{array}{c}\text { Correctly } \\
\text { identified }(\%)^{\mathrm{b}}\end{array}$ & $\begin{array}{l}\text { Indeterminate, not } \\
\text { identified }(\%)^{c}\end{array}$ & $\begin{array}{c}\text { Incorrectly } \\
\text { identified }(\%)^{\mathrm{d}}\end{array}$ & $\begin{array}{c}\text { Ambiguously } \\
\text { identified (\%) }\end{array}$ \\
\hline \multicolumn{6}{|l|}{ Microbe identification in vitro } \\
\hline Armillaria gallica & 13 & $100.0(98.0)$ & 0.0 & 0.0 & 0.0 \\
\hline A. ostoyae & 18 & $100.0(99.2)$ & 0.0 & 0.0 & 0.0 \\
\hline A. tabescens & 11 & $100.0(98.4)$ & 0.0 & 0.0 & 0.0 \\
\hline Ceratocystis fagacearum & 18 & $100.0(99.7)$ & 0.0 & 0.0 & 0.0 \\
\hline Cryphonectria parasitica & 5 & $100.0(97.9)$ & 0.0 & 0.0 & 0.0 \\
\hline Hericium erinaceus & 9 & $100.0(98.2)$ & 0.0 & 0.0 & 0.0 \\
\hline \multicolumn{6}{|l|}{ Plant host identification in vivo } \\
\hline Acer rubrum & 10 & $100.0(99.5)$ & 0.0 & 0.0 & 0.0 \\
\hline Carya illinoensis & 10 & $100.0(98.7)$ & 0.0 & 0.0 & 0.0 \\
\hline C. tomentosa & 10 & $100.0(99.7)$ & 0.0 & 0.0 & 0.0 \\
\hline Carpinus caroliniana & 10 & $100.0(99.5)$ & 0.0 & 0.0 & 0.0 \\
\hline Platanus occidentalis & 10 & $100.0(97.8)$ & 0.0 & 0.0 & 0.0 \\
\hline Populus deltoides & 10 & $100.0(99.2)$ & 0.0 & 0.0 & 0.0 \\
\hline Prosopis glandulosa & 10 & $80.0(79.8)$ & 20.0 & 0.0 & 0.0 \\
\hline Quercus alba & 10 & $100.0(99.7)$ & 0.0 & 0.0 & 0.0 \\
\hline Q. falcata & 10 & $100.0(98.4)$ & 0.0 & 0.0 & 0.0 \\
\hline Q. marilandica & 10 & $100.0(99.0)$ & 0.0 & 0.0 & 0.0 \\
\hline Q. nigra & 10 & $100.0(99.3)$ & 0.0 & 0.0 & 0.0 \\
\hline Salix nigra & 10 & $100.0(99.1)$ & 0.0 & 0.0 & 0.0 \\
\hline Taxodium distichum & 10 & $100.0(98.7)$ & 0.0 & 0.0 & 0.0 \\
\hline Sassafras albidum & 10 & $100.0(98.9)$ & 0.0 & 0.0 & 0.0 \\
\hline Ulmus crassifolia & 10 & $100.0(98.7)$ & 0.0 & 0.0 & 0.0 \\
\hline \multicolumn{6}{|l|}{ Disease diagnosis in vivo } \\
\hline \multicolumn{6}{|l|}{ Oak wilt in $Q$. fusiformis } \\
\hline
\end{tabular}

${ }^{a}$ Microbes identified in vitro consisted of cultures in sample tubes on standardized growth media. Plant host identifications and disease diagnoses were determined from sapwood tree core samples.

${ }^{b}$ Percentage of unknown samples identified correctly. Values in parentheses indicate the mean global class distribution percentage attributed to the correct identification category for that sample type.

${ }^{\mathrm{c}}$ Unidentified samples resulted from a global class distribution that had $<70 \%$ ownership in any one global class.

${ }^{\mathrm{d}}$ Misidentified samples indicate that the identity was incorrectly attributed to the wrong global class.

e Ambiguous identifications indicate that separate samples of the same type were determined to have majority ownership in different global classes.

${ }^{\mathrm{f}}$ Signature files of healthy nonhosts controls, consisting of sapwood cores of healthy hardwood species immune to $C$. fagacearum infection, were excluded from the oak wilt-live oak reference database, resulting in the inability to identify the aroma signatures of these analyte (odor) species. 
and $O$. plurianulatum with QFs of 15 to $20(P<0.001)$. The greatest difference was found between $O$. piceae and $O$. plurianulatum. Three principal components of the aroma signatures (PC 1 to 3 ) accounted for $89.8 \%$ of the variability in the analysis.

A similar comparison in all possible combinations using threedimensional PCA of headspace volatiles from sapwood cores of live oaks in various levels of infection by $C$. fagacearum demonstrated how relatedness of sample volatiles could be used as an indication of infection status for the purpose of disease diagnosis (Table 7). Healthy and inoculated control categories were samples of known origin (infection status) used as a reference against unknown samples. No significant difference or a close relationship was found between EASPs of nonsymptomatic and healthy controls. However, moderately significant differences were found between nonsymptomatic and weakly symptomatic samples, healthy controls and weakly symptomatic samples, inoculated and strongly symptomatic samples, and weakly and moderately symptomatic samples. Strong significant differences were determined between samples at different levels of infection and symptomatic categories within the extremes of the infection range. Highly significant differences were found between nonsymptomatic and strongly symptomatic and between healthy controls and strongly symptomatic samples at opposite ends of the infection range. The three principal components of aroma signatures accounted for $88.3 \%$ of the variability in the analysis.

Three-dimensional PCA of EASPs of sapwood volatiles also differentiated healthy from wetwood-infected cores of cottonwood for diagnosis of bacterial wetwood infections in standing trees and in unprocessed logs at the lumber mill (Fig. 3). Although the aroma map of healthy and infected samples indicated a somewhat diffuse distribution for both aroma classes based on a two-dimensional view, the analysis indicated a strong significant difference between these aroma classes $(\mathrm{QF}>6.0)$. The difference and clustered distributions of these aroma classes were more apparent when observed by rotating the map graphic in threedimensional space using the analysis software. The three principal components (PC 1 to 3) for each aroma class accounted for $98.8 \%$ of the variability in the analysis and were plotted as Eigen values on the $y, x$, and $z$ axis, respectively, expressed as a percentage. The PC value for each axis indicated the proportion of the difference explained by that PC. The relatively high percentage of

TABLE 7. Three-dimensional principal component analysis of headspace volatiles released from live oak sapwood tree cores at various levels of infection by Ceratocystis fagacearum, the oak wilt fungus

\begin{tabular}{llc}
\hline Analyte 1 & Analyte 2 & QF significance \\
\hline Nonsymptomatic & Healthy control & 0.81 \\
& Inoculated control & $7.82^{* *}$ \\
& Strongly symptomatic & $9.26^{* * *}$ \\
& Weakly symptomatic & $2.53^{*}$ \\
Healthy control & Moderately symptomatic & $5.10^{* *}$ \\
& Inoculated control & $7.74^{* *}$ \\
& Strongly symptomatic & $9.05^{* * *}$ \\
& Weakly symptomatic & $2.49^{*}$ \\
Inoculated control & Moderately symptomatic & $5.02^{* *}$ \\
& Strongly symptomatic & $2.59^{*}$ \\
& Weakly symptomatic & $3.50^{* *}$ \\
Strongly symptomatic & Moderately symptomatic & $4.02^{* *}$ \\
& Weakly symptomatic & $4.72^{* *}$ \\
Weakly symptomatic & Moderately symptomatic & $5.09^{* *}$ \\
& Moderately symptomatic & $2.41^{*}$
\end{tabular}

${ }^{\mathrm{a}} \mathrm{QF}=$ quality factor. Discrimination between analytes (aroma classes) was significant at the following levels: *,**, and *** indicate $P<0.05,0.01$, and 0.001 , respectively. A QF value of 2.0 indicates a significant discrimination at $\approx P=0.05$. The percentages of the total variance, accounting for the variability explained by each orthogonal principal component $(\mathrm{PC})$, are as follows: $\mathrm{PC} 1=63.66 \%, \mathrm{PC} 2=14.92 \%$, and PC $3=9.73 \%$, representing the $x, y$, and $z$ axis of the aroma map, respectively. difference (89.1\%), accounted for by the first two PCs (PC 1 and 2 ) in this analysis, was a good indication that a significant degree of difference was determined between aroma classes without the need to specify rigorous discrimination parameters for neural-net training in the construction of the reference library.

SMA of related bacteria. The relatedness of volatile metabolite mixtures produced in the culture headspace of five related phytopathogenic bacteria, formerly placed in the genus Erwinia, provided further indications of a correlation between the chemical relatedness (metabolic pathways) of microbial species and their taxonomic relatedness. Comparisons of these five species in all possible combinations using two-dimensional SMA indicated a low significant difference between Brenneria quercina and Erwinia amylovora, and between $B$. quercina and Pectobacterium carotovora (=Erwinia carotovora), with Euclidean distance (ED) values of $<1.5$ implying distinct species, but a fairly close relationship (Table 8). A higher level of significant difference was indicated between $B$. quercina and E. chrysanthemi, between B. quercina and Pantoea ananatis, between E. amylovora and E. chrysanthemi, and between E. amylvora and Pectobacterium carotovora. Similar differences were observed between $E$. chrysanthemi and Pantoea ananatis, between E. chrysanthemi and Pectobacterium carotovora, and between Pantoea ananatis and $P$. carotovora. These comparisons indicating greater differences $($ ED $>2.0)$ suggest that the analytes come from more distantly related species, perhaps supporting the separation into different genera $(P<0.01)$. The highest level of difference (ED > 3.0) was determined between E. amylovora and Pantoea ananatis, indicating that these species are most distantly related or least related among the species tested $(P<0.001)$.

Factors affecting instrument performance and analyses. Instrument operation was most affected by sample air RH. Sensors were overloaded by excess moisture in the sample. Most problems with excessive moisture were controlled by setting the reference air at $4 \%$ to maintain a relatively dry carrier stream with minimal impact on sensor sensitivity. Maintaining low referenceair relative humidity assured positive sensor responses in most cases because any additional moisture added to the analyzed

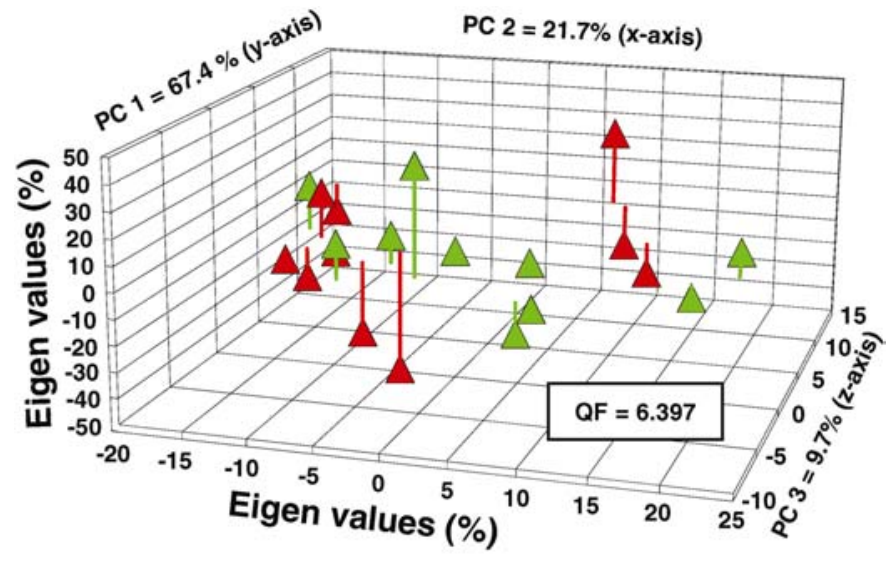

\section{$\triangle=$ Healthy cottonwood $\triangle=$ Infected cottonwood}

Fig. 3. Principal component (PC) analysis of electronic aroma signature patterns differentiating headspace volatiles released from healthy and wetwoodinfected sapwood cores of cottonwood (Populus deltoides) for disease diagnosis. Eigen values, describing the amount of variance captured in the data of each individual PC axis, were calculated by decomposing the covariance or correlation matrix representing the data. The correlation matrix was a scaled version of the covariance matrix such that every individual element in the covariance matrix is divided by the product of the standard deviations of the two covarying quantities to obtain the percentage Eigen values. The PC value for each axis indicates the proportion of the difference explained by that PC; $\mathrm{QF}=$ quality factor. 
headspace came from the sample. Effective control of reference air RH by the instrument humidity control device required that sample hydration was maintained properly. Samples such as tree cores that became too dry during cryostorage resulted in negative (below baseline) responses for all sensors throughout data acquisition. Negative responses were corrected by rehydrating the sample and air-drying immediately prior to data acquisition. Sensor response intensities also were affected by sample mass and preparation, equilibration time and temperature, and reference air quality. Sample mass and equilibration times affected the concentration of headspace volatiles. Standardizing sample preparation and equilibration methods controlled the sample size releasing volatiles and headspace accumulation. Reference air prefilters provided assurances of air quality introduced into the sampling chamber. Instrument precision was very high when these controls were strictly maintained. The accuracy of analytical determinations and disease diagnoses were improved for in vivo sampling by taking samples from several diseased parts of the plant.

\section{DISCUSSION}

The electronic nose is a chemical-sensing device containing aroma-reactive detectors capable of producing a digital fingerprint of volatiles released from any source. Conductive polymer sensor arrays take advantage of differential responses of different conducting plastics (within each sensor) to various chemical species in the sample headspace by producing a unique EASP specific to the analyte mixture. The multisensor array provides an output response pattern analogous to a combination lock that reflects the collective responses of all sensors in the array. However, unlike a combination lock, the sensor outputs are in continuous values. The pattern-recognition algorithms in the analysis software compare signature patterns stored in the reference library to those of unknown samples to look for similarities and differences in these patterns. The differences are expressed digitally as numerical values that are compared in matrix format. The algorithms assign distributions of similar elements found in PCs of the sample that are in common with known patterns in the reference library and make a determination of identity based on that distribution. Unlike gas chromatography, there is no stationary phase or retention time involved because the response of each sensor is based on the collective effect of the entire mixture of compounds in the headspace on electrical-resistance changes generated by adsorption of analytes to the sensor. Sensor adsorption is determined by the specific affinity of unique polymers in each sensor, the specificity of chemical types, quantities, and molar ratios of chemicals present in the sample mixture. Thus, using CPA avoids the need to extract specific components of microbial cells such as nucleic acids or cell wall fatty acids for microbial identifications (23), although known reference strains are required in the development of reference libraries.

CPA is a relatively new EAD technology that offers considerable potential for a wide range of applications in plant pathology from disease diagnosis and pathogen and host identification in vitro to detection and identification of plant pathogens in plant tissues as well as mixed infections, toxic metabolites (toxins), and pesticides (unpublished data). Some advantages of CPA include short analysis time (run time 90 to 220 s), high level of precision, sensitivity of detection, control of sample discrimination specificity, flexibility of sensor array selection, and custom database capabilities. Our results indicate the reliability of CPA as a diagnostic tool for plant pathology, and demonstrate an advantage over other types of diagnostic methods in that CPA methods rarely identify unknown samples incorrectly or ambiguously when an aroma class category exists for the unknown in the reference library. The absence of false positives and ambiguous determinations with CPA assures that a sample will be identified either correctly or unsuccessfully. Another significant advantage of CPA is the capability of distinguishing between sources of sample mixtures containing the exact same chemical components, but with different molar ratios of those components.

We have developed methods for the application of CPA as a new diagnostic tool for the detection and identification of diseases caused by plant-pathogenic bacteria and fungi. These methods have been used to acquire reproducible, unique EASPs useful for the identification of plant-pathogenic microbes in pure culture, the identification of host tissues, and for disease diagnosis in excised infected plant tissues. These results demonstrate the efficacy of CPA as a means of identifying individual phytopathogenic prokaryotic and eukaryotic microbes by the unique mixtures of volatile metabolites they produce in vitro and in host tissues. This technology also was used to identify the plant species from which host tissue were collected. Theoretically, it should be possible to detect obligate parasites in vivo such as viroids, viruses, nematodes, and protozoa that produce abnormal and foreign volatiles in diseased tissues or cause alterations in the types of volatile metabolites produced in diseased tissues from those found in healthy tissues. This has been the basis for the recent use of CPA by the medical diagnostics industry in the detection of cancers, ulcers, and urinary tract and upper respiratory infections in human and animal tissues $(28,44)$. Identification of EASPs indicative of specific combinations of microbial species or strains present in a sample is possible with specialized reference libraries. CPA also offers the potential for discriminating specific mixtures of pathogens and specific host-microbe combinations within host tissues. Finally, the rapid analysis possible with this technology could prove useful in the detection of plant and human pathogens for homeland security applications as real-time identifications become feasible using portable EAD devices (37).

Three-dimensional PCA of EASPs of sapwood volatiles effectively differentiated healthy from bacterial wetwood-infected cores of cottonwood for diagnosis of this disease (infection status) in standing trees. The application is potentially useful in commercial lumber production by allowing the early detection of wetwood logs coming into the lumber mill. Early detection of wetwood could mitigate economic losses by reducing or precluding lumber degrades during the drying process through adjustments in lumber drying schedules. CPA also was used effectively to determine the infection status of live oaks suspected of being infected with the oak wilt pathogen. The occurrence of unknown elements in aroma profiles of strongly symptomatic tissue could have been due to volatiles released by saprophytic microbes into necrotic diseased tissues. However, weakly symptomatic plants in early stages of oak wilt disease development did not have saprophytic contamination due to the absence of necrotic sapwood tissues. Artificially inoculated tissue tended to be overwhelmed by the

TABLE 8. Two-dimensional Sammon mapping analysis of headspace volatiles released from cultures of five bacterial species, formerly placed in the genus Erwinia, growing on standardized NBY growth medium

\begin{tabular}{llc}
\hline Analyte 1 & Analyte 2 & $\begin{array}{c}\text { Euclidean } \\
\text { distance }\end{array}$ \\
\hline Brenneria quercina & Erwinia amylovora & $1.45^{*}$ \\
& E. chrysanthemi & $2.68^{* *}$ \\
& Pantoea ananatis & $2.10^{* *}$ \\
E. amylovora & Pectobacterium carotovora & $1.14^{*}$ \\
& E. chrysanthemi & $1.91^{* *}$ \\
E. chrysanthemi & Pantoea ananatis & $3.27^{* * *}$ \\
& Pectobacterium carotovora & $1.71^{* *}$ \\
Pantoea ananatis & Pantoea ananatis & $2.35^{* *}$ \\
\hline
\end{tabular}

a Discrimination between analytes (aroma classes) was significant at the following levels: *,**, and *** indicate $P<0.05,0.01$, and 0.001 , respectively. The two-dimensional Sammon mapping model used in this analysis was significant at $P<0.002$. 
higher inoculum density, resulting in faster colonization by the pathogen that inhibited growth of competing saprophytes in necrotic tissues. Some occasional low-level misidentifications and ambiguous identifications may have resulted from the collection and analysis of poor or nonrepresentative tissue samples from the host. This demonstrates why it is important to take diseased tissue samples from several locations on the plant in order to increase confidence in the results.

The Aromascan A32S instrument, like most other conductive polymer instruments, was sensitive to sample size (total amount of organic volatiles present) and differences in $\mathrm{RH}$ between the sample and reference gases. Control of sample gas RH to within a specific range $(\leq 2 \%)$ above reference air $\mathrm{RH}$ was needed to yield the best analytical results. High detector sensitivity to moisture and certain polar compounds, particularly carboxylic acids, can cause problems in methods development for different types of samples. Dry samples had to be rehydrated in order to facilitate volatilization when building headspace to avoid negative signal outputs from the sensor array. Specificity in detection was controlled by the number of iterations used (training duration), confidence level, and other parameters selected during neural-net training. Thus, it was possible to detect differences in strains of microbial species if high specificity was used, but this could result in indeterminations if reference library specificity is set too high. Some limitations of CPA included the inability to identify individual chemical species within complex mixtures of metabolic analytes from microbes, to make reliable quantitative determinations (only semiquantitative), and the time requirement for building head space volatiles prior to analyses. Signal intensity generally was proportional to the quantity of volatiles present, but not predictably quantifiable. Sample preparation variability and time requirements for headspace building have been largely eliminated by the use of autosamplers.

Several factors affected quality, stability, and uniformity of runs during data acquisition. Sampling methods had a large impact on uniformity of signal output from the sensor array. Static sampling provided more uniform and stable data output than dynamic stripping and equilibration sampling because it avoided the dilution of headspace volatiles (increasing sensitivity) and precluded perturbations of sampling air that caused temporal variability in sample concentration during the run. Instrument architecture was modified so that reference air could be vented during sample introduction to avoid dilution effects. Samples were introduced from a closed sampling bottle without reference air introduction to maintain uniform sample concentrations during data acquisition. Consistency and repeatability of results between instruments were once a problem due to differences in sensor coating thickness, requiring calibration of sensor arrays, and correction of data between individual instruments. This problem has been resolved by improved sensor manufacturing methods that control coating thicknesses to a high tolerance. Some additional ways of improving discrimination and increasing accuracy of determinations include: (i) taking more than one sample from different parts of the plant and taking samples over time to confirm infection status, (ii) using category-specific reference libraries (developed for specific host-pathogen combinations), (iii) increasing neural-net training specificity for recognition and increasing the number of elements allowed in error before unknown identity is declared, (iv) tweaking discrimination parameter values, and (v) putting more strains into the reference library to better represent the range of strains encountered. It is very important that reference libraries are constructed using strains that originate from the geographical area where unknown samples are to be collected. Strain variability in different geographical areas can have considerable effects on resulting EASPs. Thus, attempts to identify strains of a pathogen at a location geographically distant from the area where reference strains were taken (to create the reference library) can result in ambiguous or even incorrect determinations.
There are considerable opportunities for customization of CPA for a large diversity of different sample types and applications. One such area is in the availability of a wide selection of sensor types from which a customized sensor array can be designed. The general-use sensor array used in this study was more useful for distinguishing bacteria than fungi because of the production of a greater diversity of metabolites and more oxidized secondary metabolites by prokaryotes. For example, the new OSGP28 sensor array has fewer sensors (only 28), but is designed to better discriminate the volatiles of microbes despite the lower number of sensor elements. Bacteria tend to produce a greater quantity of more oxidized metabolites (aldehydes and carboxylic acids) whereas fungi produce more reduced compounds (alcohols, ketones, and esters). This information can be used in selecting sensor arrays used for detecting specific groups of microbes. The flexibility of a selected sensor array can be further improved by selecting which sensors will be used for the analysis. Specific sensors in the array can be turned off when they do not provide significant usefulness in the discrimination. In this way, it is possible to refine the sensor array to limit it to the fewest number of sensors that will provide effective discrimination for each microbial class. However, a specific sensor combination used for identification of unknowns must be consistent with the sensors used in building the reference library. Control of the sensors being used in an analysis is useful in the development of cheaper portable field units with fewer sensors, in which only those sensors needed for discrimination are used in each specific application. This capability is essential for the miniaturization of hardware and electronics necessary in portable units. There also is flexibility in development of standardized culture media used in database development in reference libraries. It was possible to very slightly modify standardized culture media to allow the growth of certain microbes requiring specific growth factors without significantly altering the aroma signature. For example, NBY medium was amended with $1.5 \%$ (wt/vol) mannitol for culture of endosymbiotic Rhizobium leguminosarum biovars to be distinguished from pathogenic biovars. Database files also may be averaged or added together, if desired, to more precisely specify sample components being analyzed.

New emerging technologies already are providing means of improving on EAD to allow rapid discrimination of individual chemical species within aroma mixtures. Instruments are being developed that combine EAD with optical sensors (46), fast gas chromatography integrated with surface acoustic wave sensors, and programmable gate arrays instead of sensor arrays (42). These technologies will have the capability of producing recognizable high-resolution visual images of specific vapor mixtures containing many different chemical species, as well as quantifying concentrations and identifying all compounds present in the mixture.

\section{ACKNOWLEDGMENTS}

We thank D. Gross (Texas A\&M University, College Station) for some of the bacterial strains used in this study; J. Bruhn (University of Missouri, Columbia) for Armillaria strains; F. Tainter (Clemson University, Clemson, SC), D. Ingram (Mississippi State Agricultural and Extension Service, Starkville), and D. Appel (Texas A\&M University) for other fungal strains; and T. Leininger and N. Schiff for manuscript reviews.

\section{LITERATURE CITED}

1. Abe, H., Kanaya, S., Takahashi, Y., and Sasaki, S. I. 1988. Extended studies of the automated odour-sensing system based on plural semiconductor gas sensors with computerized pattern recognition techniques. Anal. Chim. Acta 215:155-168.

2. Aishima, T. 1991. Discrimination of liqueur aromas by pattern recognition analysis of responses from a gas sensor array. Anal. Chim. Acta 243:293-300.

3. Bartlett, P. N., Elliott, J. M., and Gardner, J. W. 1997. Electronic noses and their applications in the food industry. Food Technol. 51:44-48. 
4. Bartnicki-Garcia, S. 1970. Cell wall composition and other biochemical markers in fungal phylogeny. Pages 81-103 in: Phytochemical Phylogeny. J. B. Harborne, ed. Academic Press, New York.

5. Bruhn, J. N., Wetteroff, J. J., Jr., Mihail, J. D., Kabrick, J. M., and Pickens, J. B. 2000. Distribution of Armillaria species in upland Ozark Mountain forests with respect to site, overstory species composition and oak decline. Eur. J. For. Pathol. 30:43-60.

6. Davide, F. A. M., Natale, C. D., and D'Amico, A. 1995. Self-organizing sensory maps in odour classification mimicking. Biosens. Bioelectron. 10:203-218.

7. Di Natale, C., Brunink, J. A. J., and Bungaro, F. 1996. Recognition of fish storage time by a metalloporphorins-coated QMB sensor array. Meas. Sci. Technol. 7:1103-1114.

8. Di Natale, C., David, F. A. M., D’Amico, A., Sberveglieri, G., Nelli, P., Faglia, G., and Perego, C. 1995. Complex chemical pattern recognition with sensor array: The discrimination of vintage years of wine. Sens. Actuators 25:801-804.

9. Dodd, G., and Persaud, K. C. 1982. Analysis of discrimination mechanisms in the mammalian olfactory system using a model nose. Nature 299:352-355.

10. Egashira, M. 1997. Functional design of semiconductor gas sensors for measurement of smell and freshness. Pages 1385-1388 in: Proc. Int. Conf. Solid-State Sensors and Actuators. IEEE, Chicago.

11. Egashira, M., and Shimizu, Y. 1993. Odor sensing by semiconductor metal oxides. Sens. Actuators 14:443-446.

12. Freund, M. S., and Lewis, N. S. 1995. A chemically diverse conducting polymer-based electronic nose. Proc. Natl. Acad. Sci. USA 92:26522656.

13. Gardner, J. W. 1991. Detection of vapours and odours from a multisensor array using pattern recognition: Principal component and cluster analysis. Sens. Actuators 4:109-115.

14. Gardner, J. W., Craven, M., Dow, C., and Hines, E. L. 1998. The prediction of bacterial type and culture growth phase by an electronic nose with a multi-layer perception network. Meas. Sci. Technol. 9:120-127.

15. Gardner, J. W., and Shurmer, H. V. 1992. Odour discrimination with an electronic nose. Sens. Actuators 8:1-11.

16. Hanaki, S., Nakamoto, T., and Moriizumi, T. 1996. Artificial odor recognition system using neural network for estimating sensory quantities of blended fragrance. Sens. Actuators 57:65-71.

17. Hatfield, J. V., Neaves, P., Hicks, P. J., Persaud, K. C., and Tavers, P. 1994. Toward an integrated electronic nose using conducting polymer sensors. Sens. Actuators 18:221-228.

18. Hobbs, P. J., Misselbrook, T. H., and Pain, B. F. 1995. Assessment of odours from livestock wastes by a photoionization detector, an electronic nose, olfactometry, and gas chromatography-mass spectrometry. J. Agric. Eng. Res. 60:137-144.

19. Keller, P. E., Kouzes, R. T., Kangas, L. J., and Hashem, S. 1995. Transmission of olfactory information for telemedicine. Chapter 27. Pages 168172 in: Interactive Technology and the New Paradigm for Healthcare. K. Morgan, R. M. Satava, H. B. Sieburg, R. Matteus, and J. P. Christensen, eds. IOS Press and Ohmsha, Amsterdam.

20. Kowaiski, B. R., and Bender, C. F. 1972. Pattern recognition: A powerful approach to interpreting chemical data. J. Am. Chem. Soc. 94:5632-5639.

21. Lonergan, M. C., Severin, E. J., Doleman, B. J., Beaber, S. A., Grubbs, R. H., and Lewis, N. S. 1996. Array-based vapor sensing using chemically sensitive, carbon black-polymer resistors. Chem. Mater. 8:2298-2312.

22. Meyerhoff, M. E. 1993. Polymer membrane-based ion-, gas-, and bioselective potentiometric sensors. Biosens. Bioelectron. 8:1-38.

23. Moss, C. W., Dees, S. B., and Guerrant, G. O. 1974. Gas liquid chromatography of bacterial fatty acids with a fused silica capillary column. J. Clin. Microbiol. 28:80-85.

24. Mullis, K. B., and Faloona, F. A. 1987. Specific synthesis of DNA in vitro via a polymerase-catalyzed chain reaction. Methods Enzymol. 155:335350 .

25. Mullis, K., Faloona, F., Scharf, S., Saiki, R., Horn, G., and Erlich, H. 1986. Specific enzymatic amplification of DNA in vitro: The polymerase chain reaction. Cold Spring Harbor Symp. Quant. Biol. 51:263-273.

26. Nanto, H., Sokooshi, H., and Kawai, T. 1993. Aluminum-doped ZnO thin film gas sensor capable of detecting freshness of sea foods. Sens. Actuators 14:715-717.

27. Ouellette, J. 1999. Electronic noses sniff out new markets. Ind. Physic. 5:26-29.
28. Parry, A. D., Chadwick, P. R., Simon, D., Oppenheim, B. A., and McCollum, C. N. 1994. Detection of $\beta$-haemolytic streptococcal infections by analysis of leg ulcer odour. J. Woun. Car. 3:134-137.

29. Pelosi, P., and Persaud, K. C. 1988. Gas sensors: Towards an artificial nose. Pages 361-381 in: Sensors and Sensory Systems for Advanced Robotics. P. Dario, ed. Springer-Verlag, Berlin.

30. Persaud, K. C. 1992. Electronic gas and odor detectors that mimic chemoreception in animals. Trends Anal. Chem. 11:61-67.

31. Persaud, K. C., Bartlett, J., and Pelosi, P. 1993. Design strategies for gas and odour sensors which mimic the olfactory system. Pages 579-602 in: Robots and Biological Systems: Towards a New Bionics? P. Dario, G. Sandini, and P. Aebisher, eds. Springer-Verlag, Berlin.

32. Persaud, K. C., Khaffaf, S. M., Hobbs, P. J., Misselbrook, T. H., and Sneath, R. G. 1996. Application of conducting polymer odor sensing arrays to agricultural malodour monitoring. Pages 249-253 in: Proc. Int. Conf. Air Pollut. Agric. Operations. Midwest Plan Service, Kansas City, MO.

33. Persaud, K. C., Qutob, A. A., Travers, P., Pisanelli, A. M., and Szyszko, S. 1994. Odor evaluation of foods using conducting polymer arrays and neural net pattern recognition. Pages 708-710 in: Olfaction and Taste XI. K. Kurihara, N. Suzuki, and H. Ogawa, eds. Springer-Verlag, Tokyo.

34. Pisanelli, A. M., Qutob, A. A., Travers, P., Szyszko, S., and Persaud, K. C. 1994. Applications of multi-array polymer sensors to food industries. Life Chem. Rep. 11:303-308.

35. Saiki, R. K., Scharf, S., Faloona, F., Mullis, K. B., Horn, G. T., Erlich, H. A., and Arnheim, N. 1985. Enzymatic amplification of $\beta$-globin genomic sequences and restriction site analysis for diagnosis of sickle cell anemia. Science 230:1350-1354.

36. Sasser, M. 1990. Identification of bacteria by gas chromatography of cellular fatty acids. MIDI Tech. Note 101, Newark, DE.

37. Schaad, N. W., Frederick, R. D., Shaw, J., Schneider, W. L., Hickson, R., Petrillo, M. D., and Luster, D. G. 2003. Advances in molecular-based diagnostics in meeting crop biosecurity and phytosanitary issues. Annu. Rev. Phytopathol. 41:305-324.

38. Shirley, S. G., and Persaud, K. C. 1990. The biochemistry of vertebrate olfaction and taste. Sernm. Neurosci. 2:59-68.

39. Shurmer, H. V. 1990. An electronic nose: A sensitive and discrimination substitute for a mammalian olfactory system. Intern. Electric. Eng. Proc. 137:197-204.

40. Shurmer, H. V., Gardner, J. W., and Chan, H. T. 1989. The application of discrimination techniques to alcohols and tobacco using tin oxide sensors. Sens. Actuators 18:359-369.

41. Simon, J. E., Hetzroni, A., Bordelon, B., Miles, G. E., and Charles, D. J. 1996. Electronic sensing of aromatic volatiles for quality sorting of blueberries. J. Food Sci. 61:967-972.

42. Staples, E. J. 2000. Electronic nose simulation of olfactory response containing 500 orthogonal sensors in 10 seconds. Pages 307-313 in: Proc. 1999 IEEE Ultrasonics Frequency Control and Ferroelectrics Symp. Lake Tahoe, CA.

43. Strassburger, K. J. 1996. Electronic nose evaluation in the flavor industry: It really works! Food Test. Anal. 2:22-37.

44. Thaler, E. R., Kennedy, D. W., and Hanson, C. W. 2001. Medical applications of electronic nose technology: Review of current status. Am. J. Rhinol. 15:291-295.

45. Vidaver, A. K. 1967. Synthetic and complex media for rapid detection of fluorescence of phytopathogenic pseudomonads: Effect of the carbon source. Appl. Microbiol. 15:1523-1524.

46. White, J., Kauer, J. S., Dickinson, T. A., and Walt, D. R. 1996. Rapid analyte recognition in a device based on optical sensors and the olfactory system. Anal. Chem. 68:2191-2202.

47. Winquist, F., Hornsten, E. G., Sundgren, H., and Lundstrom, I. 1993. Performance of an electronic nose for quality estimation of ground meat. Meas. Sci. Technol. 4:1493-1500.

48. Wilson, A. D., and Lester, D. G. 1997. Use of an electronic-nose device for profiling headspace volatile metabolites to rapidly identify phytopathogenic microbes. (Abstr.) Phytopathology 87(suppl.):S116.

49. Wilson, A. D., and Lester, D. G. 1998. Application of aromascan analysis to diagnose oak wilt in live oaks. (Abstr.) Phytopathology 88(suppl.):S97.

50. Yea, B., Konishi, R., Osaki, T., and Sugahara, K. 1994. The discrimination of many kinds of odor species using fuzzy reasoning and neural networks. Sens. Actuators 45:159-165.

51. Yim, H. S., Kibbey, C. E., Ma, S. C., Kliza, D. M., Liu, D., Park, S. B., Torre, C. E., and Meyerhoff, M. E. 1993. Polymer membrane-based ion-, gas-, and bio-selective potentiometric sensors. Biosens. Bioelectron. 8:1-38. 